Revue d'histoire de l'Amérique française

ZWB REVUE D.HISTOIRE DE L'AMÉRIQUE FRANÇAISE

\title{
Les obligations du censitaire, à l'époque des Cent-Associés
}

\section{Marcel Trudel}

Volume 27, numéro 1, juin 1973

URI : https://id.erudit.org/iderudit/303230ar

DOI : https://doi.org/10.7202/303230ar

Aller au sommaire du numéro

Éditeur(s)

Institut d'histoire de l'Amérique française

ISSN

0035-2357 (imprimé)

1492-1383 (numérique)

Découvrir la revue

Citer cet article

Trudel, M. (1973). Les obligations du censitaire, à l'époque des Cent-Associés. Revue d'histoire de l'Amérique française, 27(1), 3-41.

https://doi.org/10.7202/303230ar d'utilisation que vous pouvez consulter en ligne.

https://apropos.erudit.org/fr/usagers/politique-dutilisation/ 


\title{
LES OBLIGATIONS DU CENSITAIRE, À L'ÉPOQUE DES CENT-ASSOCIÉS *
}

\author{
MARCEL TRUDel \\ titulaire de recherche \\ à l'Université d'Ottawa
}

Propriétaire absolu de la terre qu'on lui a accordée en fief, le seigneur doit en attribuer des parcelles à ceux qui veulent s'établir chez lui, mais cette attribution n'est ni une transmission de biens qui serait la suite d'un acte de vente, ni une cession qui aurait le caractère d'une donation pure et simple; elle est concession, c'est-à-dire un acte par lequel un seigneur cède une parcelle de terre dont il se réserve la propriété éminente et sur l'exploitation de laquelle il perçoit des droits.

Les tout premiers actes de concession donnent seulement une brève description de la terre concédée, sans même énumérer les conditions de la concession: encore en 1635, l'acte de concession de 40 arpents à Mathieu Huboust, ne va pas au-delà d'une localisation géographique. L'énumération des servitudes commencerait seulement en 1636. Nous croyons en trouver le premier exemple dans un acte du 27 août 1636, en vertu duquel Jean Côté reçoit 6 arpents dans la banlieue de Québec: l'acte précise que Côté devra défricher sa terre, souffrir les chemins que les Cent-Associés jugeront nécessaires, payer chaque année un denier de cens par arpent; on le dispense, toutefois, de ce dernier devoir pendant les dix premières années. Après ce premier acte, le patron devient définitif; l'acte de concession va désormais présenter ses éléments selon un ordre généralement respecté: le seigneur qui concède la terre, le concessionnaire, les dimensions de la terre concédée, le taux du cens et de la rente, et les autres servitudes. Certes, dans les tout débuts, il arrive que ces servitudes ne soient pas inscrites dans l'acte de concession: ainsi, lorsque Guillaume Couture obtient une terre dans

* Cette étude est extraite d'un ouvrage en préparation, Les débuts du régime seigneurial au Canada. Au cours de cet article, nous utilisons les sigles suivants :
AHD-Q Archives de l'Hôtel-Dieu de Québec
APC Archives publiques du Canada
ASQ Archives du Séminaire de Québec
EO Edits, ordonnances royaux (éd. 1854)
PTIO Papier terrier de la Compagnie des Indes occidentales, 1667- 1668 (éd. P.-G. Roy)
RAPQ Rapport de l'archiviste de la province de Québec. 
la seigneurie de Lauson, le 15 mai 1647 , la concession est faite "aux charges Que Messieurs de la compagnie ordonneront"1; fréquent dans le cas de concession par les Cent-Associés (l'acte de ratification viendra compléter), ce procédé est, toutefois, rare chez les autres seigneurs.

Pour observer ces rapports entre le censitaire et le seigneur, voyons d'abord un contrat de concession, bien typique de ceux qu'on accorde sur la côte de Beaupré et que l'on peut considérer, en quelque sorte, comme le contrat-type qui sera utilisé dans les autres seigneuries. Après avoir décrit la terre qu'il concède à Jean Doyon, le 20 juillet 1652, au nom de la Compagnie de Beaupré, le gouverneur Jean de Lauson ajoute les conditions suivantes:

moyennant que le dit Doyon s'est obligé de s'y établir dans la présente année et y avoir feu et lieu, ou autre pour lui, et de payer au jour de Saint-Martin d'hiver de la présente année et de continuer à l'avenir par chacun an, pour chacun arpent de front sur le dit grand fleuve Saint-Laurent, vingt sols tournois à la recette des seigneurs de Beaupré de rente seigneuriale, douze deniers de cens et la dîme au curé lorsque le cas y écherra, et pour la dite concession deux chapons vifs en l'hôtel seigneurial du dit Beaupré ou autres lieux qui seront désignés dans la dite seigneurie, les dits cens et rentes portant lots et ventes, saisine et amendes, selon la Coutume de la Prévôté et Vicomté de Paris quand le cas y écherra; ne pourra le dit Doyon chasser ni pêcher que sur et vis-à-vis la présente concession sans le gré et consentement de ses voisins; à la charge de laisser entre ses tenants de chacun côté un chemin de quinze pieds de large et autant le long du fleuve pour servir de chemin à la navigation; et en cas que les seigneurs de Beaupré fassent construire des moulins dans la seigneurie de Beaupré, le dit Doyon s'oblige d'apporter ses grains moudre en icelui; les prés le long du dit fleuve, communs, qu'il pourra faire faucher sur la présente concession au préjudice de tous autres et de plus, pour éviter à procès et entretenir amitié entre les tenants du dit Doyon, en plus il sera obligé de clore ses terres au fur et à mesure qu'il les fera défricher, à faute de ce faire il ne pourra prétendre aucun dommage et intérêts pour les délits et dégâts que pourront faire les bestiaux de ses voisins $[\ldots]^{2}$.

1 Acte de concession du 10 juil. 1790 (copie collationnée de l'acte de 1637, minutier Deschenaux).

2 Acte de concession du 20 juil. 1652 (minutier Auber). A cause de la longueur de ce texte cité, nous avons jugé opportun d'en moderniser l'orthographe. 
a - le cens

Numériquement la moins importante des obligations, le cens est un impôt annuel qu'il faut payer au seigneur et qui demeure comme "la marque de la directe Seigneurie" 3 ; plutôt qu'un revenu utile, c'est une "marque d'honneur" que l'on doit à son seigneur ${ }^{4}$; par le cens, celui qui se fait concéder une terre en roture (c'est-à-dire sans qualité, à la différence d'une terre en seigneurie), reconnaît que le seigneur garde la propriété éminente sur la terre concédée: le censitaire, ainsi appelé parce qu'il est soumis au cens, n'est pas rigoureusement propriétaire du sol concédé: il n'en est que le concessionnaire, et c'est par le cens qu'il avoue son infériorité à l'égard de la propriété. Le cens, de ce fait, est le fondement même des droits du seigneur sur la terre qu'il concède sans qualité; selon la Coutume de Paris, c'est parce qu'il a droit de cens que le seigneur peut exiger d'autres droits "qui en sont une suite" 5 ou, comme le veut la formule si souvent répétée dans le Terrier de 1667-1668, "ledit cens portant lods et ventes, saisines et amendes"

"Marque de seigneurie", il n'appartient qu'au seigneur de l'imposer; celui qui tient une terre à cens "ne peut rebailler à cens, parce qu'il n'est Seigneur de fief, \& et que cens ne peut être mis sur cens" ?. C'est l'erreur commise par Jean Guion du Buisson: il s'était fait concéder en 1659, dans le fief du Sault-au-Matelot, un emplacement pour lequel il payait un cens de 6 deniers au seigneur Couillard, son beau-père; or Guion du Buisson en reconcède une partie à son frère Claude, en exigeant un cens d'un denier; le juge Chartier de Lotbinière lui rappellera en 1668 que le cens n'appartient qu'aux seigneurs ${ }^{8}$. Il est arrivé aux Ursulines de découvrir la même erreur dans un contrat qu'elles avaient accordé ${ }^{9}$. Si un censitaire sous-arrente

$3 \mathrm{La}$ Coutume de Paris définit le cens: "prestation annuelle qui se paye tous les ans par le possesseur d'un heritage tenu à cette charge, au Seigneur censier, c'est-à-dire au Seigneur d'un Fief duquel releve cet heritage"; cette prestation annuelle est "la marque de la directe Seigneurie" (Claude de Ferrière, Nouveaw commentaire sur la Coutume de Paris (éd. 1728), I: 143).

4 Dans un arrêt de Tracy et de Talon, en 1667, on lit: Sa Majesté "peut créer en leur faveur [les officiers] quelques droits de cens ou censives peu considérables, qui soient plutôt des marques d'honneur que des revenus utiles" (EO, II: 34).

5 Ferrière, op. cit., I: $143 \mathrm{~s}$.

6 PTIO, passim.

7 Ferrière, op. cit., I: 143.

8 PTIO, 256s.

$9 \mathrm{La}$ veuve d'Ailleboust déclare en 1667 un emplacement d'un demiarpent; or, dans le contrat de concession, il était dit qu'un cens était payable 
sa terre, en tout ou en partie, il ne peut percevoir qu'une rente, et le locataire, en proportion de ce qu'il occupe, fournira sa quote-part dans le paiement du cens au seigneur.

La "marque de seigneurie" qu'est le cens peut s'exprimer autrement que par une somme d'argent. Cela se produit à l'égard d'une terre concédée en franc-alleu. Car, de même qu'il y a seigneurie en franc-alleu, c'est-à-dire sans suzerain et, par conséquent, sans devoirs, de même il y a terre concédée en francalleu par le seigneur: une terre en franc-alleu se trouve libérée de toute "reconnoissance de directe Seigneurie" et de toute obligation pécuniaire à l'égard du seigneur ${ }^{10}$; toutefois, dans certains cas, le seigneur peut, en accordant le franc-alleu, se réserver un devoir de gratitude. C'est le cas des Ursulines de Québec, à qui les Cent-Associés ont accordé des terres en francalleu dans Québec: ils exigent, en retour, outre un aveu tous les vingt ans, une messe solennelle pour "la gloire et pour l'honneur de la France et celle de l'establissement de la colonie", messe à laquelle seront invités le gouverneur, son lieutenant et les habitants de Québec ${ }^{11}$. C'est aussi le cas de Simon Denys de La Trinité, à qui les Jésuites ont accordé, "eu Egard a sa Condition Et Grande famille", une augmentation extrêmement généreuse de terre; cette augmentation sera libre de devoirs pécuniaires (à l'exception des lods et ventes), mais Denys de La Trinité devra, chaque année, le jour de la fête du Saint-Sacrement, porter une couronne de fleurs à la chapelle des Jésuites ${ }^{12}$. Jean Gloria sera aussi dispensé de payer le cens en argent pour

aux Ursulines; le procureur des religieuses vient déclarer qu'elles ne "prétendent avoir aucun droit de cens" et que le dit cens mentionné dans le contrat, "a esté une surprise et une erreur" (PTIO, 99). De plus, dans la concession d'un emplacement à Jean Normand par les Ursulines, le 15 mai 1663 (minutier Audouart), il est dit que Normand doit aux Ursulines une rente et un denier de cens: les Ursulines n'étant pas seigneuresses de cette terre, le notaire aurait dû indiquer que le cens est payable aux Cent-Associés. 10 "Le Franc-aleu est un heritage qui n'est sujet à aucun devoirs \& droits Seigneuriaux, tant honorifiques comme la foi \& hommage, que pecuniaires, comme cens, quint, relief ou autres, en reconnoissance de directe Seigneurie; c'est pourquoi il est ainsi appellé, quasi liberum \& solutum ab omnibus juribus, \& il est dit alleu, quasi sine laudatione, parce que qui eo jure proedia possidet nullum habet autorem vel dominum, quem laudare possit vel debeat" (Ferrière, op. cit., I: 138).

11 Cette obligation sera transférée à la Compagnie des Indes occidentales, "afin de mouvoir nos seigneurs présentement propriétaires de la dite seigneurie dominante de ce pays d'avoir pour elles la mesme volonté"; à partir de 1667, ce jour sera le premier lundi du mois de juin, jour de l'assemblée générale des seigneurs (PTIO, 97).

12 Acte du 10 août 1652 (copie collationnée par Chambalon), ASQ, Seigneuries, 1: 30. Ce jour-là, les Jésuites concèdent d'abord 7 arpents sur 24, puis ils en étendent la profondeur jusqu'à 4 lieues. 
une terre relevant de la seigneurie de Saint-François; cette fois, au lieu de fleurs, c'est deux œufs frais qu'il doit présenter à son seigneur Jean Bourdon, le 27 décembre, fête de saint Jean l'Evangéliste ${ }^{13}$.

En principe, le tarif du cens est fixé dès l'acte de concession, mais, du temps des Cent-Associés, il y a dans les titres bien du désordre, dont témoigne l'enquête de la Compagnie des Indes occidentales en 1667-1668. Des censitaires ignorent le tarif de leur cens, parce qu'il n'était pas inscrit dans leurs titres de concession; ce qui se produit couramment dans les titres accordés au nom des Cent-Associés: leur agent leur laissait le soin de fixer ce tarif, lors de la ratification; ou bien le concessionnaire a négligé de faire ratifier son titre, ou l'acte de ratification a été perdu: nous connaissons là-dessus une dizaine d'exemples ${ }^{14}$. Ou bien c'est le vendeur d'un emplacement qui néglige d'en informer l'acquéreur: en 1668, Pierre Disy déclare, que, pour l'emplacement qu'il a acheté de Bertrand Fafard en 1660 , le contrat d'acquest ne fait aucune mention de cens; le juge en fixera un, séance tenante ${ }^{15}$. D'autres censitaires ne savent à quel cens ils sont soumis, parce qu'ils ne peuvent retrouver des titres antérieurs à 1663; ainsi, à l'occasion d'un contrat de vente, François Boivin avait remis son titre au notaire Becquet, "des mains duquel il ne l'a pu retirer" ${ }^{16}$; le titre de l'emplacement de Jacques Sevestre est demeuré parmi les papiers de l'ancien gouverneur d'Argenson ${ }^{17}$; le marchand Claude Charron détient un emplacement concédé en ou avant 1656; il en avait confié le titre à Charles de Lauson-Charny (ex-gouverneur par intérim), en vue de le faire ratifier à Paris,

13 Aveu et dénombrement de la seigneurie de Saint-François, 3 nov. 1668 (minutier Duquet). Nous ne sommes pas sûr, toutefois, que Gloria ait déjà une terre dans cette seigneurie en 1663: nous l'avons laissée de côté dans notre inventaire de juin 1663.

14 C'est le cas des concessions suivantes: terre de 351 arpents consédée en 1649 à Godefroy de Normanville, aux Trois-Rivières (PTIO, 295); emplacement concédé à Seigneuret, aux Trois-Rivières, en 1650 (PTIO, 306) ; terre de 44 arpents, concédée en 1644 à François Marguerie, des TroisRivières (PTIO, 302s.) ; emplacement concédé à Nicolas Marsolet, à Québec, en 1651 (PTIO, 130) ; emplacement concédé à Guillaume Gaultier dit Lachesnaye, à Québec, en 1657 (PTIO, 226s.); terre de 3 arpents, concédée à René Robineau de Bécancour en 1657 (PTIO, 354); emplacement de la basse-ville de Québec, concédé à Eustache Lambert, par le gouverneur d'Argenson (PTIO, 174) ; emplacement concédé à Bertrand Fafard, aux Trois-Rivières, avant 1660 (PTIO, 363s.) ; emplacement concédé à Pierre Lepetit, à Québec, vers le même temps (PTIO, 121) ; terre concédée à Etienne Vien avant 1663 (PTIO, 324).

15 PTIO, 362s.

16 PTIO, 205.

17 PTIO, 67s. 
mais, Lauson-Charny ayant été capturé par les Espagnols, le titre n'a pas été retrouvé ${ }^{18}$; celui du marchand François Perron a disparu dans un incendie ${ }^{19}$; d'autres titres se sont perdus ${ }^{20}$. Un autre est devenu illisible, "pour avoir esté gasté par l'eau" 21.

Enfin, il y a les censitaires qui, en 1667-1668, n'occupent encore que sur une simple promesse verbale de concession, des portions de sol qu'on leur a attribuées avant 1663; c'est le cas, par exemple, de Nicolas Bonhomme: encore en 1667, il occupe une terre sur la rivière Saint-Charles, en vertu seulement d'une promesse verbale que lui a faite le gouverneur d'Argenson ${ }^{22}$; comme c'est le cas de Claude Volant dit SaintClaude: pour l'emplacement qu'on lui a accordé aux Trois-Rivières vers 1656 , il n'a pas encore de titre écrit en $1668{ }^{23}$.

Nous disposons quand même d'éléments suffisants pour déterminer le tarif du cens dans les seigneuries ou dans les terres qui relèvent des Cent-Associés:

RÉGION DE QuébeC

Beaupré

12 deniers par arpent de front

le tarif est le même dans les arrière-fiefs, sauf à Beaulieu, où les terres sont toutes petites: il y est de 3 deniers pour l'ensemble d'une concession, qu'elle soit de 2 ou de 4 arpents de front

Beauport

12 deniers par arpent de front

sauf pour la terre de Cadieu ( 3 arpents sur 20), où le cens est de 24 deniers par arpent de front; le tarif de 12 deniers par arpent de front est aussi en vigueur dans les arrière-fiefs

Notre-Dame-des-Anges

2 deniers par concession

sauf pour la terre de Trefflé ( $21 / 2$ arpents sur 40$)$, dont le cens est d'un denier pour toute la concession; les terres de Denys de La Ronde (71/2 arpents sur 40), de Crevier (4 arpents sur 24), de Normand ( 2 arpents sur 30 ), de Lambert ( 2 arpents sur 40 ) et de Roy ( 2 arpents sur 40 ) sont à 3 deniers pour toute la concession

Saint-Joseph

Saint-Ignace

18 PTIO, 152.

19 PTIO, 32.

20 PTIO, 133, 159, 243, 325, 326; pour éviter un procès à la suite de la perte d'un titre, on accorde un nouveau titre (acte du 23 avril 1657, minutier Audouart).

21 PTIO, 326.

22 PTIO, 156s.

23 PTIO, 341s.
2 deniers par concession

2 deniers par concession 
banlieue de Québec

6 deniers par arpent de superficie sauf pour les terres de Nepveu (21/2 arpents sur 9), de Sevestre $(4,7$ arpents sur 10), de Lemire (11/2 arpent sur 10), de Juchereau de Maure (96 arpents), de Juchereau de La Ferté (9 arpents), de Joseph Hébert (2,8 arpents sur 71/2), de Françoise Hébert (3,3 arpents sur 6,9), d'Abraham Martin (terre de 1639: 20 arpents) et des frères Pinguet (3 arpents sur 10), qui sont à un denier par arpent; la terre de Lelièvre et Marette (11/2 arpent) est à 9 deniers pour le tout; la terre d'Abraham Martin (12 arpents) est à 12 deniers; toutes ces terres, sauf celle de 12 arpents qui a été concédée à Martin en 1650, ont été concédées de 1635 à 1639

\section{Coulonge}

Saint-François estimation fondée sur un seul cas connu

6 deniers par arpent de superficie

3 deniers par arpent de superficie

2 deniers par concession

sauf pour la terre de Migneron ( $11 / 2$ arpent sur 20), qui est à un denier; celle de Liénart (2 arpents sur 29,2) est à 12 deniers; dans l'arrière-fief Sainte-Ursule, le tarif est aussi de 2 deniers par concession

Gaudarville

6 deniers par arpent de superficie sauf pour la terre de Damours (4 arpents sur 121/2), à un denier par arpent de superficie

\section{Lauson}

12 deniers par arpent de front

sauf pour la terre de Charles de Lauson-Charny ( 8 arpents sur 40), qui était à un denier seulement pour toute la concession, Lauson-Charny étant le fils de Jean de Lauson (cette terre fait partie en 1663 des 15 arpents sur 40 qui appartiennent à Thomas Lefebvre)

\section{RÉGION DES TROIS-RIVIÈRES}

Cap-de-la-Madeleine

île de la Trinité

l'Islet

île Saint-Christophe

banlieue des Trois-Rivières
2 deniers par concession

6 deniers par arpent de superficie

12 deniers par arpent de superficie

2 deniers par concession

6 deniers par arpent de superficie sauf pour les terres de David ( 2 arpents sur 24), de Fafard (2,4 arpents sur 17, 4 arpents sur 10 et 53 arpents), d'Aubuchon ( 2 arpents sur 10), d'Ameau ( 5 arpents sur 20), de Pierre Lefebvre (21 arpents sur 84), qui sont à un denier par arpent de superficie; celle de Leneuf de La Poterie (0,78 arpent), à 2 deniers par arpent de superficie; celles de Leneuf de $\mathrm{La}$ Poterie (2,3 arpents sur 21,6; 300 arpents; 4 arpents sur 25, un arpent sur 10), de Leneuf du Hérisson $(2,3$ arpents sur 21,6) à 3 deniers par arpent de superficie 
MontréAL

Montréal (campagne)

3 deniers par arpent de superficie

sauf pour la terre de d'Ailleboust des Muceaux (86 arpents) et de LeMoyne (90 arpents), qui sont à 5 sols (ou 60 deniers) par concession.

Comment expliquer ces exceptions à l'intérieur d'une même seigneurie ou des terres qui relèvent des Cent-Associés ? Comme les critères discriminatifs ne sont nulle part décrits, nous pourrions tenter de les fonder sur la qualité du concessionnaire: ce critère peut expliquer, par exemple, que Lauson-Charny, fils du seigneur de Lauson, ne paie à son père qu'un denier de cens pour toute sa concession de 320 arpents, quand les voisins paient 12 deniers par arpent de front; que Damours des Chaufours, d'origine noble, ne paie dans Gaudarville qu'un denier par arpent, alors que les autres en paient six; que Leneuf de La Poterie, aussi noble, ne paie que 2 ou 3 deniers par arpent, quand les autres sont à un tarif de 6 deniers; toutefois, ce critère ne s'applique qu'à certains cas: en diverses occasions, on favorise également gens de qualité et petites gens. L'époque de la concession pourrait aussi expliquer un certain nombre d'exceptions: nous notons, en particulier, que des concessions faites de 1635 à 1639 sont à un tarif de faveur de seulement un denier par arpent de superficie, mais ce tarif de faveur ne s'applique pas à toutes les concessions de la même époque. Peut-être la situation d'une terre pourrait-elle, en certains cas, tenir lieu de critère ? $\mathrm{Du}$ reste, les exceptions sont peu nombreuses.

Eu égard à leur tarif, nous pouvons répartir en 4 groupes les terres soumises au cens:

\section{2 deniers par concession}

Notre-Dame-des-Anges (Jésuites)

Saint-Joseph (veuve de Guillaume Couillart)

Saint-Ignace (Hôtel-Dieu de Québec)

Sillery (Sauvages chrétiens sous la direction des Jésuites)

Cap-de-la-Madeleine (Jésuites)

île Saint-Christophe (Jésuites)

12 deniers par arpent de front

Beaupré (Compagnie de Beaupré)

Beauport (Robert Giffard)

Lauson (Jean de Lauson, fils)

3 deniers par arpent de superficie

Montréal (Société de Notre-Dame, puis Sulpiciens) 
6 deniers par arpent de superficie banlieue de Québec (Cent-Associés)

Coulonge (veuve de d'Ailleboust)

Gaudarville (Cent-Associés, puis veuve de Lauson de La Citière)

île de la Trinité (Cent-Associés)

banlieue des Trois-Rivières ${ }^{24}$ (Cent-Associés).

Nous avons donc ici quatre tarifs différents, que nous énumérons en allant du plus faible au plus élevé:

2 deniers par concession

12 deniers par arpent de front

3 deniers par arpent de superficie

6 deniers par arpent de superficie.

Si nous appliquons ces tarifs à la moyenne des terres en censive, nous obtenons pour 1663 le tableau suivant:

\begin{tabular}{|c|c|c|c|c|c|c|c|}
\hline & \multicolumn{3}{|c|}{$\begin{array}{l}\text { dimensions } \\
\text { de la terre moyenne } \\
\text { (en arpents) }\end{array}$} & \multicolumn{4}{|c|}{ cens perçu sur la terre moyenne } \\
\hline & 3,7 & x 93,2: & 344,8 & 44,4 deniers: & & 3 s. & $8 \mathrm{~d}$. \\
\hline Beauport & 3,1 & x 57,3: & 177,6 & 37,2 deniers: & & $3 \mathrm{~s}$. & \\
\hline Notre-Dame-des-Anges & & x 69,7: & 209,1 & 2 deniers: & & & \\
\hline Saint-Joseph & 1,7 & x 34,4: & 58,5 & 2 deniers: & & & \\
\hline banlieue de Québec & 3 & x 9,7: & 29,1 & 174,6 deniers: & & $14 \mathrm{~s}$. & 7 \\
\hline Sillery & 2,3 & x 24,5: & 56,4 & 2 deniers: & & & $2 \mathrm{~d}$. \\
\hline arville & 3,6 & $\times 24,8$ : & 89,3 & 535,8 deniers: & 2 liv. & $4 \mathrm{~s}$. & $8 \mathrm{~d}$. \\
\hline Lau & 4,7 & x 39,1: & 183,3 & 56,4 deniers: & & $4 \mathrm{~s}$. & \\
\hline Cap-de-la-Madeleine & 1,9 & x 29,8: & 56,6 & 2 deniers: & & & 2 \\
\hline la Trinité & & & 50 & deniers: & 1 liv. & $5 \mathrm{~s}$. & \\
\hline$\underset{R}{b a n l}$ & 4,2 & & & & 1 liv & & \\
\hline Montréal & 1,7 & x 16,3: & 27,7 & 83,1 deniers: & & $6 \mathrm{~s}$. & \\
\hline
\end{tabular}

De ces cens moyens, quatre nous surprennent à première vue par leur tarif élevé: dans la banlieue de Québec, 14 sols 7 deniers; dans l'île de la Trinité, 1 livre 5 sols; dans la banlieue des Trois-Rivières, 1 livre 19 sols 1 denier; dans la seigneurie de Gaudarville, 2 livres 4 sols 8 deniers. Il s'agit, dans chaque cas, de terres qui ont été concédées par les Cent-Associés: or les terres qui relèvent directement d'eux, ne sont soumises qu'au cens et non aux rentes, alors que les terres qui relèvent des autres seigneurs doivent payer à la fois un cens et

24 Nous ne tenons pas pour exception l'Islet (à l'embouchure du SaintMaurice), soumis à 12 deniers de cens pour le tout: cette île, qui relève des Cent-Associés, aurait un arpent seulement, mais on a pu, à cette époque, calculer qu'elle avait un arpent ou deux. 
une rente ${ }^{25}$. A Montréal, le cens moyen ( 6 sols 11 deniers) nous paraît élevé, lui aussi, mais les terres de l'île ne sont encore qu'à cens, ce qui place quand même les Montréalistes dans une situation avantageuse. Le cens le plus faible, 2 deniers par concession, est celui de la seigneurie de Saint-Joseph (qui appartient aux Couillart) et celui des seigneuries jésuites: NotreDame-des-Anges, Sillery, Cap-de-la-Madeleine et île Saint-Christophe; en ces cas, le cens n'est vraiment tout juste qu'un symbole de propriété éminente du seigneur.

Dans les villes, le cens est plus difficile à observer. Certes, aux Trois-Rivières, ville-damier, les emplacements sont soumis à un tarif uniforme: un denier de cens chacun, sauf quatre exceptions ${ }^{26}$; à Montréal, qui tend à devenir ville-damier, il y a aussi uniformité, au moins dans les cas connus: le tarif serait de 5 sols par arpent, mais nous connaissons au moins une exception: l'arpent de Pierre Gadois, concédé en 1643, est à 3 deniers seulement l'arpent, quand ses concitoyens qui reçoivent des emplacements en même temps que lui, sont à 5 sols (ou 60 deniers) l'arpent. A Québec, non seulement à cause de l'émiettement du sol, mais surtout à cause de son émiettement très irrégulier, nous n'arrivons pas à déterminer un tarif de base. La charge d'un emplacement de Charles Amiot est "douze deniers de cens par dix pieds remettant et quarante cinq pieds à quatorze sols six deniers" 27 ; celui de la veuve de Duplessis-Kerbodot est à 12 deniers de cens, "par chaque dix pieds" et, lors de la ratification, on écrit que le cens est de 60 sols ${ }^{28}$ : dans le premier cas, le cens aurait été de 9 livres 14 sols 4 deniers; dans le second, il se trouverait réduit à 3 livres, soit environ 4 deniers par dix pieds. Il y a grande variété de tarifs dans les autres emplacements: elle va de un denier jusqu'à 7 sols ou 84 deniers ${ }^{29}$; deux terres qui relèvent de la Fabrique ont

25 La plupart des terres de Gaudarville ont été concédées par les CentAssociés (donc, à cens seulement), avant l'érection de la seigneurie, en 1652; nous ignorons si le seigneur de 1652 a émis de nouveaux titres pour modifier le cens et introduire la rente.

26 L'emplacement de l'Algonquin Pachiriny (concédé en 1649) est à 3 deniers pour le tout; un emplacement de Pierre Boucher (concédé en 1655) est à 3 deniers; un autre lui est accordé à 6 deniers (emplacement à la campagne: donc, tarif de la campagne); un emplacement de Leneuf de La Poterie est à 30 sols (ou 11/2 livre): c'est que Leneuf de La Poterie y exploite un moulin à vent.

27 PTIO, 46; emplacement de 45 pieds sur 40 (soit 50 toises), situé près de la fontaine Champlain, aux abords de la basse-ville.

28 PTIO, 206s.; emplacement de 108 pieds sur 18 (ou 54 toises).

29 Nous trouvons les tarifs suivants: $1,2,3,4,6,9$ deniers; $1,2,3$ sols; 2 sols 6 deniers; 4 sols 6 deniers; 4 sols 8 deniers; 5 sols; 7 sols. Pour les 
un tarif de 2 deniers par perche ${ }^{30}$. Comme, en plusieurs cas, on compte la rente par perche, peut-être le cens est-il, lui aussi, calculé par perche ? En 1754, il y aurait, semble-t-il, un tarif régulier de 5 sols 6 deniers par emplacement ${ }^{31}$, mais nous ne voyons pas que ce tarif, qui ne peut être qu'un tarif de base, puisse nous aider à clarifier la situation de 1663 .

Même si nous connaissions exactement à combien de cens chaque emplacement urbain et chaque terre rurale sont soumis, il faudrait tenir compte des sursis qui sont accordés. Il y eut de ces sursis, dès les débuts, au moins de la part des Cent-Associés. Ainsi, Guillaume Hébert, à qui on concède 22 arpents en 1639 , est dispensé du cens pendant les dix premières années ${ }^{32}$ : c'est un cadeau de 11 sols par année, ou de $51 / 2$ livres en dix ans. Il en est de même pour les 160 arpents concédés à Pierre Legardeur de Repentigny en 1639 et en $1640^{33}$ : don de 40 livres en dix ans. Pour la terre de 93 arpents qu'on lui concède en 1647, Bertrand Fafard est dispensé de payer son cens d'un denier par arpent, pendant six ans, à compter du jour de la prise de possession ${ }^{34}$. Pierre Lefebvre, qui est soumis au même tarif pour sa terre de 21 arpents sur 84, ne paiera le cens qu'à partir du moment où la terre sera en valeur ${ }^{35}$ : c'était lui faire un cadeau annuel de 71/4 livres par an; mais cette terre, concédée en 1647 sur la rive sud du fleuve, n'est pas encore en valeur en 1663; on fait à Lefebvre la même faveur pour une terre de 100 arpents, concédée en 1656 et soumise à 6 deniers de cens par arpent ${ }^{36}$ : cadeau annuel de $21 / 2$ livres.

Il faut aussi tenir compte des arrérages. Ils paraissent nombreux du temps des Cent-Associés, si l'on en juge par le Papier terrier de 1667-1668. Sans retenir ici ceux qui déclarent ne pas savoir à combien le cens était fixé, nous relevons au moins 18 noms de censitaires qui n'ont pas payé aux Cent-Associés le cens qui leur était dû; parmi ces retardataires, il y a des gens im-

2 arpents que Simon Denys de La Trinité possède dans le Mont-Carmel et dans la falaise, le tarif est de un sol par arpent.

30 La perche de superficie mesure 324 pieds carrés. L'emplacement de Louis Chapelain (père), 14 perches et un tiers, est soumis à 2 sols de rente par perche, soit 1 livre 8 sols 8 deniers; et à 2 deniers de cens, probablement par perche.

31 EO, II: 421 (Bigot y parle de cens et rentes, mais nous croyons qu'il ne s'agit, en fait, que de cens, la censive du roi n'étant pas soumise à la rente).

32 PTIO, 151.

33 PTIO, 181.

34 PTIO, 298s.

35 PTIO, 321.

36 PTIO, 328. 
portants: les Ursulines, Simon Denys de La Trinité, Leneuf de La Poterie, les frères Pinguet; sept ont été condamnés à une amende, plutôt symbolique, de 5 sols, dont les Ursulines ${ }^{37}$. Ce désordre dans la perception du cens par les Cent-Associés sur leurs terres de Québec et de la banlieue, n'est pas un fait unique; nous le retrouvons à la fin du régime français: enquête faite, l'intendant Bigot constate en 1754 que le tarif des trois-quarts des emplacements de Québec (relevant alors du roi) est inconnu et "à régler"; il ordonne qu'on procède au recouvrement de 29 années d'arrérages et "qu'à l'avenir le dit recouvrement se fera tous les dix ans" ${ }^{38}$. Nous ignorons si, du temps des Cent-Associés, on a aussi laissé traîner le paiement du cens dans les seigneuries.

Enfin, en nous fondant sur la liste que nous avons établie dans notre Terrier du Saint-Laurent, essayons, bien que de nombreuses données fassent défaut, de déterminer d'une façon au moins approximative, les sommes que la perception du cens pouvait rapporter ou que les seigneurs pouvaient s'attendre à recueillir sur les terres rurales pour l'année 1662:

\begin{tabular}{|c|c|c|c|c|}
\hline & $\begin{array}{l}\text { total des } \\
\text { terres }\end{array}$ & $\begin{array}{c}\operatorname{somn} \\
d u\end{array}$ & ppro & $\begin{array}{l}\text { mative } \\
\text { evoir }\end{array}$ \\
\hline REGGTON DE QUUÉBEC & & livres & sols & deniers \\
\hline $\begin{array}{l}\text { Beaupré } \\
\text { Compagnie de Beaupré }\end{array}$ & & & & \\
\hline $\begin{array}{l}\text { Compagnie de Beaupré } \\
\text { arrière-fief Repentigny-Villiers }\end{array}$ & 92 & 22 & 6 & $\begin{array}{l}6 \\
6\end{array}$ \\
\hline arrière-fief Repentigny & 6 & & 13 & 6 \\
\hline arrière-fief Lothainville & 8 & 1 & 2 & \\
\hline arrière-fief Argentenay & 2 & & 7 & \\
\hline arrière-fief Charny-Lirec & 65 & 10 & 4 & 6 \\
\hline arrière-fief Beaulieu & 10 & & 2 & 3 \\
\hline Beauport & 10 & 1 & $1 \overline{2}$ & \\
\hline arrière-fief Beaumarchais & 1 & & 2 & \\
\hline Notre-Dame-des-Anges & 20 & & 6 & 6 \\
\hline $\begin{array}{l}\text { Saint-Joseph } \\
\text { Saint-Ignace }\end{array}$ & 5 & & & 10 \\
\hline Saint-Ignace & 1 & & & 2 \\
\hline banlieue de Québec & 62 & 46 & 3 & 10 \\
\hline Coulonge & 5 & 3 & 9 & \\
\hline Saint-François & 1 & & 2 & \\
\hline Sillery & 30 & & 6 & 5 \\
\hline arrière-fief Sainte-Ursule & 2 & & & \\
\hline Gaudarville & 14 & 27 & 1 & 2 \\
\hline Lauson & 25 & 5 & 14 & 4 \\
\hline arrière-fief Sainte-Anne & 3 & & 7 & \\
\hline
\end{tabular}

37 Voir PTIO, 19, 46, 47, 94, 189, 192, 194, 199, 216, 227, 228, 236, 241, 245s., 248, 249, 250, 255. Ont été condamnés à une amende les Ursulines, la veuve de Bascon, Mathieu Huboust dit Deslongschamps, les héritiers Larchevêque, Denis Duquet, Noël Boëssel. $38 \mathrm{EO}$, II: 421. 
REGION DES TROIS-RIVIÈRES

\begin{tabular}{|c|c|c|c|}
\hline $\begin{array}{l}\text { Cap-de-la-Madeleine } \\
\text { ile de la Trinité } \\
\text { L'Islet } \\
\text { ile Saint-Christophe } \\
\text { banlieue des Trois-Rivières }\end{array}$ & $\begin{array}{r}40 \\
1 \\
1 \\
7 \\
51\end{array}$ & 77 & $\begin{array}{l}6 \\
5 \\
1 \\
1 \\
6\end{array}$ \\
\hline $\begin{array}{c}\text { MONTREAL } \\
\text { Montréal (campagne) }\end{array}$ & 84 & 27 & 12 \\
\hline
\end{tabular}

Ce qui produit un total de 228 livres 5 sols 6 deniers à percevoir en cens sur 560 terres rurales, soit une moyenne de 7 sols 8 deniers par terre. Ajoutons à ce total 1 livre 14 sols 9 deniers pour le cens de 49 emplacements aux Trois-Rivières, et 5 livres 19 sols pour le cens de 31 emplacements à Montréal, et nous obtenons ainsi un montant global (qui n'inclut pas les emplacements de Québec) d'au moins 235 livres 19 sols 3 deniers que les censitaires doivent verser aux seigneurs pour le cens, en 1662. Petit montant global, dont plus de la moitié (126 livres 11 sols) va aux Cent-Associés, à cause de terres de banlieue et d'emplacements urbains; leur part de cens serait encore plus élevée, si nous connaissions le tarif de base des emplacements de Québec. De toute façon, le produit du cens n'a que valeur de symbole: chaque terre rurale ne paie en 1662 que 8 sols 1 denier, l'équivalent d'une demi-livre de beurre ${ }^{39}$.

\section{$\mathrm{b}$ - la rente}

Ces terres sur lesquelles ils affirment leur propriété éminente par le cens, les seigneurs, à l'exception des Cent-Associés et de la Société de Notre-Dame, les ont concédées pour en retirer un revenu. Ce revenu est dit rente foncière non rachetable, parce que, produite par un fonds de terre, elle ne peut, selon la Coutume de Paris, être rachetée; ce qui la différencie de la rente constituée attachée à la personne et non au fonds et qui peut être liquidée; en d'autres termes, la rente foncière est perpétuelle de sa nature ${ }^{40}$.

Cette rente peut être, du temps des Cent-Associés, en argent ou en nature, ou (dans la plupart des cas) en l'un et l'autre. S'il y a paiement en nature, c'est en beurre, en blé, en blé d'Inde, mais, le plus souvent, c'est en chapons vifs ou en poules grasses,

\footnotetext{
39 En 1655 et en 1660, le beurre coûte 15 sols la livre (Audouart, actes des 30 juin 1655 et ler fév. 1660).

40 Merlin, Répertoire universel et raisonné de jurisprudence, II: art. Cens. Il faudra une loi spéciale du Parlement en 1854, pour permettre aux censitaires de racheter cette rente foncière.
} 
le seigneur se réservant, toutefois, le choix entre l'animal et l'équivalent en argent: 20 sols, d'ordinaire, pour un chapon vif. Il arrive exceptionnellement que la rente soit versée en prières: pour la terre de 15 arpents sur 40 que Charles de Lauson-Charny cède à l'Hôtel-Dieu en 1657, les religieuses devront chaque année, le 29 octobre, et à perpétuité, célébrer un service solennel à la mémoire de l'épouse de Lauson-Charny, décédée le 30 octobre $1656^{41}$.

Voici les tarifs que nous avons relevés:

RÉGION DE QUÉBEC

Beaupré

$$
\begin{aligned}
& 20 \text { sols } \\
& \text { par arpent de front }
\end{aligned}
$$

2 chapons vifs pour la terre ou 40 sols, au choix du seigneur

le cas le plus fréquent est celui de 2 chapons pour l'ensemble de la terre, qu'elle soit de 3, 4, 5 ou 6 arpents de front; nous relevons une terre de 20 arpents de front qui est soumise à l'obligation de 4 chapons;

il en est de même dans les arrière-fiefs, sauf que, dans RepentignyVilliers, Chesnaye doit donner 3 chapons pour sa terre de 3 arpents de front; l'arrière-fief Beaulieu, dont les terres sont beaucoup plus petites qu'ailleurs, a ses conditions particulières: dans le cas de Blanquet, la rente est de 25 sols par arpent de front; en d'autres cas, c'est 20 sols par arpent de front ou même seulement 10 sols; chaque terre doit fournir 3 chapons

Beauport

20 sols par arpent de front

2 chapons vifs pour la terre ou 40 sols, au choix du seigneur

le chapon est à 15 sols en 1637 (un cas), en 1645 (un cas) et en 1649 (3 cas); dans un acte de concession, on a remplacé chapons par poules grasses

Notre-Dame-

des-Anges

$$
\begin{array}{ll}
\text { de } 20 \text { à } 40 \text { sols } & 1,2,3 \text { ou } 4 \text { chapons pour la } \\
\text { par arpent de front } & \text { terre }
\end{array}
$$

6 terres sont à 20 sols par arpent de front; 4 à 25 sols, 7 à 30 sols (une terre de 1649,3 terres de 1658,2 terres de 1659 , une terre de 1661 ); une terre de 1658 est à 40 sols; pour une terre de 3 arpents sur 24, Chalifour doit s'acquitter de 60 sols, de 2 chapons et de 2 livres de beurre; nous comptons un chapon pour une terre de 2 arpents de front et pour une terre de 5 arpents de front; 2 chapons pour des terres dont le front à $11 / 2,2,3$ ou 4 arpents; une terre de $71 / 2$ arpents de front est soumise à 3 chapons; une terre de 3 arpents de front, à 4 chapons, comme aussi une terre de 4 arpents de front; pour sa terre de $21 / 2$ arpents de front, Trefflé doit 5 chapons; nous connaissons un cas (terre de 2 arpents de front), où le seigneur exige 2 chapons et une poule

41 Acte de cession du 26 juin 1657, AHD-Q, Terrier des anciens titres, 47a. Ce service solennel a été célébré chaque année, jusqu'en 1930. 
Saint-Joseph

Saint-Ignace

étude d'un cas seulement
20 sols

par arpent de front

2 chapons vifs pour la terre

2 chapons vifs, en tout

banlieue de Québec

en principe, les Cent-Associés ne perçoivent pas de rente sur les terres de la banlieue de Québec ${ }^{42}$

\section{Coulonge}

Saint-François

Saint-Michel

Sillery
20 sols par arpent de front dans les deux premiers rangs; 1 sol par arpent de superficie dans le $3^{\mathrm{e}}$ rang et dans la côte Sainte-Geneviève
2 chapons vifs, en tout

2 chapons vifs, en tout

2 chapons vifs, en tout

2 chapons vifs pour la terre ou 2 poules vives

2 terres sont à 15 sols par arpent de front; les terres de la côte SaintFrançois-Xavier ( $1^{\mathrm{er}}$ rang) sont à 20 sols par arpent de front; aussi celles de la côte Sainte-Ignace ( $2^{\mathrm{e}} \mathrm{rang}$ ); celles de la côte Saint-Michel ( $3^{\mathrm{e}}$ rang) sont à 1 sol par arpent de superficie;

en général, que la terre ait 2 ou 3 arpents de front, c'est 2 chapons ou 2 poules vives, mais on rencontre aussi un chapon pour une terre d'un arpent et demi de front, et pour une terre de 3 arpents sur 20; 6 chapons pour une terre de 6 arpents de front; dans l'arrière-fief Sainte-Ursule, la rente est d'un sol par arpent de superficie; et pour chaque terre de cet arrière-fief, un chapon ou une poule

\section{Gaudarville}

les concessions, faites à l'origine par les Cent-Associés, ne comportaient pas de rente; nous ignorons s'il y a rente en 1663

Lauson

20 sols par arpent de front

2 chapons vifs pour la terre

il en est de même dans l'arrière-fief Saint-Vilmé, mais ici c'est 4 chapons pour chaque terre; les terres de Guillaume et d'André Albert (chacune de 120 arpents) sont à 15 sols par arpent de front

\section{RÉGION DES TROIS-RIVIÈRES}

\section{Cap-de-la- \\ Madeleine \\ 2 boisseaux de blé froment par terre \\ 1 chapon pour la terre} seulement deux cas de connus

42 Notons, cependant, que la terre de 8 arpents de Charles Legardeur de Tilly est soumise à une rente d'une poule. 
île de la Trinité

les concessions, faites par les Cent-Associés, ne comportaient pas de rente

l'Islet

les concessions, faites par les Cent-Associés, ne comportaient pas de rente

île Saint-Christophe un demi-boisseau de

blé par terre

banlieue des Trois-Rivières

les concessions, faites par les Cent-Associés, ne comportaient pas de rente

MONTRÉAL

Montréal (campagne)

les concessions, faites par la Société de Notre-Dame, ne comportaient pas de rente.

Les censitaires de la ville de Québec, là où ils relèvent des Cent-Associés, ne paient que le cens et non la rente, alors que les censitaires de la Fabrique de Notre-Dame lui paient en rente 2 sols par perche: la moyenne versée par emplacement en 1663 serait de 54 sols, ou 2 livres 14 sols. Point de rente à payer par les habitants du bourg des Trois-Rivières, puisque les emplacements relèvent des Cent-Associés; point de rente non plus à payer par les habitants de la ville de Montréal. Dans le village de Beauport, dont le sol relève directement du seigneur, les emplacements, presque tous d'un arpent sur dix, sont à 10 sols l'arpent de front, plus une poule grasse pour l'ensemble du lot: chaque emplacement aurait ainsi, en 1663, à payer en moyenne une rente de 27 sols 6 deniers.

Si nous voulons établir qui sont, parmi les censitaires ruraux, les mieux favorisés par rapport au tarif de la rente seigneuriale, nous avons d'abord ceux qui n'en ont aucune à payer, ce sont les censitaires des seigneuries ou régions suivantes:

QuÉBEC

banlieue de Québec

Gaudarville

île de la Trinité

LES TROIS-RIVIÈRES

l'Islet

banlieue des Trois-Rivières
21 terres

12 terres

1 terre

1 terre

56 terres 


\section{MONTRÉAL}
Montréal (campagne)
95 terres.

C'est un total de 186 terres sur les 660 du Saint-Laurent: les $28,2 \%$; plus du quart des terres n'ont donc pas de rente à payer.

Pour 9 autres terres (ce sont celles de l'île Saint-Christophe), la rente est de seulement un demi-boisseau de blé par terre, soit la somme de 20 sols, ou 1 livre ${ }^{43}$. Elle est du double (deux chapons par terre ou 2 livres) pour les 10 terres des seigneuries de Saint-Ignace, de Coulonge, de Saint-Michel et de Saint-François. Pour le reste des terres du Saint-Laurent, c'està-dire les 68,9\%, la rente annuelle est d'au moins 4 livres.

En nous fondant encore sur notre Terrier du Saint-Laurent et en laissant de côté, bien entendu, les seigneuries et régions où la rente n'a pas lieu, tentons un calcul approximatif de ce que la rente pouvait produire en 1662:

\section{Beaupré \\ Compagnie de Beaupré \\ arrière-fief Repentigny- Villiers \\ arrière-fief Repentigny \\ arrière-fief Lothainville \\ arrière-fief Argentenay \\ arrière-fief Charny-Lirec arrière-arrière-fief $\mathrm{La}$ Chevalerie arrière-fief Beaulieu \\ Beauport arrière-fief Beaumarchais \\ Notre-Dame-des-Anges 45 \\ Saint-Joseph \\ Saint-Ignace \\ Coulonge \\ Saint-François \\ Sillery arrière-fief Sainte-Ursule \\ Lauson arrière-fief Sainte-Anne arrière-fief Saint-Vilmé \\ Cap-de-la-Madeleine 46 \\ île Saint-Christophe 47 total}

$\begin{gathered}\text { total des } \\ \text { terres } \\ \text { comptes }\end{gathered}$

92

5
6
8
2
65
9
10
10
1
20
5
1
5
1
30
2
25
3
1
40
7
348

rente d

percevoir nombre de

43 Le boisseau de blé se vend 2 livres en 1662 (comptes de la Fabrique de Sainte-Anne-de-Beaupré).

44 Sous le titre chapons, nous classons aussi les poules dont il est question dans Beauport et dans Sillery. Chapons et poules sont évalués, à cette époque, à 20 sols (évaluations fixées dans les actes de concession). 45 Une rente est aussi en 2 livres de beurre; en 1655 et en 1660, le 
Pour ces 348 terres arrentées, les rentes de 1662 se monteraient donc au total de 1818 livres 2 sols, dont une partie est en nature: 654 chapons ou poules grasses, 841/2 boisseaux de blé et 2 livres de beurre; à quoi il faut ajouter un service solennel que l'Hôtel-Dieu doit à la mémoire de l'épouse de LausonCharny. Répartie sur les 348 terres, la somme globale produit une moyenne de 5 livres 4 sols 6 deniers.

Le total le plus élevé est celui que la Compagnie de Beaupré retire de 92 terres qui relèvent d'elle directement: 623 livres 3 sols; vient ensuite celui que devrait toucher Charles de LausonCharny, pour 65 terres de son arrière-fief: 334 livres 8 sols. Les Jésuites perçoivent bien un total de 387 livres 18 sols 6 deniers, mais c'est en réunissant les seigneuries de Notre-Dame-desAnges, de Sillery, du Cap-de-la-Madeleine et l'île Saint-Christophe. Tout compté ce que produit l'ensemble d'un même fief, c'est encore celui de Beaupré (côte de Beaupré et île d'Orléans réunies) qui vient en tête, et bien loin avant tous les autres: 1140 livres 11 sols, pour un total de 197 terres; rente qui inclut un troupeau impressionnant de 400 chapons vifs, soit les $61,2 \%$ de tous les chapons et poules grasses présentés en rente en 1662 .

\section{$\mathrm{c}$ - les lods et ventes}

En plus de l'imposition d'une rente, le cens entraîne la perception d'une taxe dite lods et ventes, sur une terre qui change de possesseur autrement que par succession directe; en vertu de la Coutume de Paris ${ }^{48}$, cette taxe, à payer par le nouveau possesseur dans les vingt jours qui suivent l'acquisition, est le "douzième du prix de la vente dont elle charge l'acquereur".

beurre coute 15 sols la livre (actes d'Audouart, 30 juin 1655 et 1er février 1660 ), et il est encore de 15 sols en 1665 . Nous l'incluons ici dans le total à percevoir.

${ }^{46}$ La rente est de 2 boisseaux de blé par concession, plus un chapon.

47 La rente est d'un demi-boisseau de blé par concession.

$48 \mathrm{La}$ Coutume de Paris définit les lods et ventes: "droits de vente dûs au Seigneur censier, sont de douze deniers un denier [...] Ce droit se paye au Seigneur censier in recognitionem directi domini; appellé dans plusieurs Coutumes lots \& ventes, quasi lots \& ventes, comme étant le lot ou la part \& portion que le Seigneur prend sur le prix de la vente [...] Notre Coutume regle ce droit au douzième du prix de la vente dont elle charge l'acquereur: toutefois les Seigneurs peuvent stipuler par les contrats d'accensements [c'est-à-dire de mise en censive] de plus grands droits" (Ferrière, Nouveau commentaire sur la Coutume de Paris (éd. 1728), I: 148s). Voir aussi PTIO, 94. 
Dans les actes de concession, on se contente d'écrire que la terre, le cas échéant, sera soumise aux lods et ventes, sans déterminer quel en sera le tarif; comme les concessions ont été faites en vertu de la Coutume de Paris (sauf exception pour certaines clauses), nous en déduisons que, du temps des Cent-Associés, le tarif a été au moins du douzième, et rien dans la documentation ne nous autorise à penser que des seigneurs auraient exigé davantage.

Bien des terres en censive ont changé de possesseurs, pendant la période des Cent-Associés: nous étudierons, en son lieu, l'étonnante instabilité des censitaires. Nous avons constaté que, sur 738 terres observées ${ }^{49}, 205$ ont changé de mains autrement que par voie d'héritage: ce sont les $30,5 \%$. De ces 205 terres, 146 ont fait l'objet de vente; si 86 n'ont été vendues qu'une fois, il en est 60 qui l'ont été plus d'une fois, dont une l'a été jusqu'à 9 fois ${ }^{50}$. Selon nos calculs, la taxe des lods et ventes aurait dû s'appliquer 240 fois. A-t-elle été fidèlement payée au seigneur ? Notre information ne porte malheureusement que sur les terres qui relèvent en droite ligne des Cent-Associés. Dans un cas, celui de l'Hôtel-Dieu de Québec qui achète en 1647 les terres de Marin Boucher et des frères Caumont, sur la rivière SaintCharles, les Cent-Associés font don des lods et ventes à l'acquéreur ${ }^{51}$. Dans d'autres cas, les lods et ventes restent dus aux CentAssociés, et ils le sont encore lors de l'enquête de 1667-1668: pour l'arpent et demi que l'Hôtel-Dieu a acheté de Guillaume Couillart en 1644, pour 400 livres, l'hôpital doit encore en 1668 une taxe de quelque 33 livres ${ }^{52}$; pour l'emplacement acquis d'Antoine Lebohême, en 1654, au prix de 800 livres, Loyer dit Latour doit encore en 1668, environ 66 livres pour lods et ventes $^{53}$. De ces retardataires d'avant 1663 , nous savons que certains seront mis à l'amende en 1667-1668; c'est le cas, en particulier, de Loyer dit Latour ${ }^{54}$. Dans les seigneuries, les re-

49 Dans ces 738 terres, nous comprenons 719 terres arrentées ou sousarrentées en 1663, et 19 terres qui, après avoir été concédées, ont été abandonnées et le sont encore en 1663. Nous n'avons pas tenu compte de certaines terres qui entraient difficilement dans nos statistiques: par exemple, celles qui, une fois vendues, ont été intégrées dans une terre plus grande ou ont été subdivisées; ou encore celles dont on n'a vendu qu'une partie.

5086 terres ont été vendues une fois; 40 l'ont été deux fois; 13 l'ont été trois fois; 4 , quatre fois; 2 , cinq fois; une terre a été vendue neuf fois. 51 PTIO, 161.

52 PTIO, 165s. Nos calculs sont faits sur le tarif du douzième.

63 PTIO, 182-184.

54 PTIO, 184. Le montant de l'amende n'est pas indiqué. Les Ursulines sont aussi condamnées à une amende ( 3 livres 15 sols) pour n'avoir pas acquitté les lods et ventes d'une terre achetée des héritiers d'Abraham Martin, mais il s'agit ici d'une acquisition de 1667 (PTIO, 92-94). 
tardataires ne devaient pas, non plus, manquer : la documentation ne nous en révèle presque rien ${ }^{55}$.

Comme nous l'avons fait pour les cens et rentes, essayons de déterminer ce que le droit des lods et ventes a pu rapporter aux seigneurs en 1662. Cette année-là, il n'y aurait eu que vingt-cinq actes de vente de terres rurales et 16 ventes d'emplacements; en ne retenant que les 21 terres et les 11 emplacements dont nous connaissons le prix de vente, nous pouvons construire le tableau suivant:

\begin{tabular}{|c|c|c|c|c|}
\hline \multirow[b]{2}{*}{ Beaunré } & $\begin{array}{c}\text { total des } \\
\text { prix de vente } \\
\text { livres sols deniers }\end{array}$ & \multicolumn{3}{|c|}{$\begin{array}{c}\text { total des } \\
\text { lods et ventes } \\
\text { livres sols denier }\end{array}$} \\
\hline & & & & \\
\hline Compagnie de Beaupré 56 & 3120 & 260 & & \\
\hline $\begin{array}{l}\text { arrière-fief Repentigny-Villiers } 57 \\
\text { arrière-fief Repentigny } 58\end{array}$ & 397 & 33 & 1 & 8 \\
\hline arrière-fief Repentigny 58 & ? & ? & & \\
\hline $\begin{array}{l}\text { arrière-fief Lothainville } 59 \\
\text { arrière-fief Charny-Lirec } 60\end{array}$ & 160 & 13 & 6 & 8 \\
\hline arrière-fief Charny-Lirec 60 & ? & ? & & \\
\hline $\begin{array}{l}\text { Notre-Dame-des-Anges } 61 \\
\text { Québec: ville } 62\end{array}$ & 640 & 53 & 6 & 8 \\
\hline $\begin{array}{l}\text { Québec: ville } 62 \\
\text { Québec: banlieue } 63\end{array}$ & 1020 & 85 & & \\
\hline $\begin{array}{l}\text { Québec: banlieue } 63 \\
\text { Saint-Michel } 64\end{array}$ & 60 & 5 & & \\
\hline $\begin{array}{l}\text { Saint-Michel } 64 \\
\text { Sillerv } 65\end{array}$ & 120 & 10 & & \\
\hline Sillery 65 & 424 & 35 & 6 & 8 \\
\hline arrière-fief Sainte-Ursule 66 & 120 & 10 & & \\
\hline Gaudarville 67 & 200 & 16 & 13 & 4 \\
\hline Cap-de-la-Madeleine 68 & 800 & 66 & 13 & 4 \\
\hline Ile-aux-Cochons 69 & 300 & 25 & & \\
\hline Trois-Rivières: ville 70 & 705 & 58 & 15 & \\
\hline Montréal: ville ${ }^{71}$ & 1720 & 143 & $\mathbf{5}$ & \\
\hline Montréal: campagne 72 & 25 & 2 & 1 & 8 \\
\hline
\end{tabular}

55 Le 22 octobre 1649, Barthélémy Gaudin s'engage, par une obligation, à payer en 1650, à l'arrivée des navires, un montant de 45 livres pour lods et ventes d'une terre qu'il a achetée (minutier Bermen). Le 9 janv. 1661, Jacques Milot devient propriétaire de la totalité d'une terre, dont il avait acquis une première moitié le 22 septembre 1660: Chomedey lui délivre quittance des lods et ventes, le 30 mai 1661; et le 7 juin 1661, Urbain Jetté remet à Milot 30 livres pour lods et ventes de cette même terre qu'il avait acquise avant Milot et pour laquelle Milot avait acquitté les droits (acte du 7 juin 1661, minutier Basset).

56 La terre de Robineau de Bécancour vendue à Michel Roullois, le 7 août, pour 2000 livres; la terre de François Fortin vendue à Nicolas Huot, le 9 fév., pour 1120 livres; la terre de Marin Dalleray vendue à Michel Esnault, le 22 oct., à un prix inconnu.

57 La terre de Pierre Boucher vendue à Louis Marthelot, le 29 juin, pour 50 livres, terre reconcédée à Marin Nourrice, puis revendue par lui à Jean Ouimet, le 2 oct., pour 200 livres; la terre de Robert Delaberge vendue à Charles Pouliot, le 22 oct., pour 120 livres; la terre de Noël Rose vendue à Claude Petiot, le 5 nov., pour 27 livres. 58 Une terre vendue en deux parties par François Fortin et Geoffroy Guillot, le 18 mars, dont le prix est inconnu. 59 La terre de Symphorien Rousseau vendue à Noël Rose, le 5 mars, 
La taxe de vente sur les terres et emplacements aurait donc rapporté en 1662 le total de 817 livres 10 sols. Total obtenu d'une façon théorique: à l'occasion d'aucune de ces ventes, nous n'avons la preuve que les lods et ventes ont été perçus; il se peut que des seigneurs ne les aient pas exigés, comme il se peut qu'ils aient accordé des rabais; et dans le cas des 4 arpents vendus par les Godé à la Fabrique de Notre-Dame-de-Montréal, au prix

pour 60 livres, puis revendue à Pierre Boucher, le 1er avril, pour 100 livres; la terre de Pierre Lepetit vendue à Abel Turcot, le 12 août, à un prix inconnu. $60 \mathrm{La}$ terre de Laurent Denis vendue à Charles Courtois, le 28 janv., à un prix inconnu.

61 La terre de Jean Normand vendue à Gervais Normand, le 11 juil., pour 240 livres; la terre de Mathieu Huboust vendue à Isaac Bédard, le 5 mars, pour 400 livres.

62 Un emplacement vendu par Christophe Crevier à Charles Aubert de La Chesnaye, le 3 juil., pour 700 livres; un emplacement cédé par Denis Guion à François Guion, le 3 sept., en échange d'un autre emplacement de valeur inconnue (nous ignorons, en ce cas, le montant des lods et ventes); un emplacement vendu par Bertrand Fafard à Jean Bourdon, le 13 juil., pour 320 livres.

63 Une terre de 2 arpents, vendue par Jean Millouer à Guillaume Fournier, le 15 janv., pour 60 livres.

${ }^{64} \mathrm{La}$ terre de Jacques Nourry vendue à Sébastien Langelier, le $1^{\text {er }}$ mars, pour 120 livres.

$65 \mathrm{La}$ terre de Claude Charland vendue à Pierre Pluchon, le 24 sept., pour 200 livres; la terre de Gilles d'Anjou vendue à Pierre Pinel, le 16 mars, pour 50 livres; la terre de M lle $^{\text {le }}$ Penart vendue à Jean Durand, le 15 mars, pour 174 livres.

120 livres.

${ }_{66} \mathrm{La}$ terre de Jean Neau vendue à Pierre Duval, le 18 juil., pour livres.

${ }^{67} \mathrm{La}$ terre de Gilles d'Anjou vendue à Thomas, le 24 août, pour 200 800 livres.

$68 \mathrm{La}$ terre de Jean Cusson vendue à Nicolas Gastineau, le 8 sept., pour 300 livres.

69 La terre de Jean Garnier vendue à Séverin Ameau, le 10 juil., pour

70 Un emplacement vendu par la veuve Ancquetin à Jean Bourdeau, le 12 sept., pour 135 livres; un emplacement adjugé à Maurice Poulain, le 12 août, pour 90 livres; un emplacement vendu par Nicolas Gaillou à Louis Pinard, le 15 août, pour un prix inconnu; un emplacement vendu par Jacques Ménard à Jean Denoyon, le 11 juil., pour un prix inconnu; un emplacement vendu par Guillaume Constantin à Séverin Ameau, le 7 mai, pour 425 livres; un emplacement vendu par Nicolas Gaillou à Alexandre Raoul, le 15 août, pour un prix inconnu; un emplacement vendu par Catherine Dodier à Jean Denoyon, le 29 juin, pour 55 livres.

71 Un emplacement vendu par Jean Dumets à François Piron, le 3 nov., pour 170 livres; deux emplacements vendus par André Charly à Marguerite Bourgeoys, le 6 juil., pour 600 livres; 4 arpents vendus par les Godé à la Fabrique, le 16 août, pour 800 livres; un emplacement vendu par Gilles Lauson à François Bailly, le 22 déc., pour 150 livres. 25 livres.

${ }^{72}$ La terre de Pierre Lorrain vendue à Jean Gasteau, le 8 oct., pour 
de 800 livres, nous avons tout lieu de croire que la Société de Notre-Dame, responsable de la vie religieuse de Ville-Marie, n'a pas réclamé de lods et ventes contre la Fabrique.

\section{$\mathrm{d}$ - la banalité $d u$ moulin à farine}

Outre le cens, la rente et les lods et ventes, le seigneur perçoit aussi d'autres droits qui sont d'un certain rapport.

Il jouit de la banalité du moulin, c'est-à-dire qu'à l'intérieur de la seigneurie, le monopole du moulin à farine (qu'il s'agisse de moulin à vent ou de moulin à eau) lui appartient. Du temps des Cent-Associés, ce monopole semble ne s'exercer en NouvelleFrance que par tradition seigneuriale, puisque les premiers règlements officiels sont postérieurs à $1663^{73}$; il est, en tout cas, affirmé dans tous les actes de concession, selon une formule qui varie peu d'une seigneurie à une autre; dans la seigneurie de Beaupré, la formule des premières années est généralement la suivante: "en cas que les seigneurs de Beaupré fassent construire des moulins dans la seigneurie de Beaupré, le dit Doyon s'oblige d'apporter ses grains moudre en icelui" ${ }^{74}$. En 1663, toutes les seigneuries, loin de là, n'ont pas encore leur moulin; dans le fief Beaupré, on en connaît deux: le moulin à vent de la Compagnie de Beaupré au Château-Richer, construit depuis au moins $1657^{75}$, et un autre, à eau celui-là, sur la rivière du Sault-à-la-Puce, qui serait de la même année ${ }^{76}$; dans l'arrièrefief Argentenay (à l'extrémité nord-est de l'île d'Orléans), il y avait eu un moulin vers 1656, mais il n'est plus en activité en $1663^{77}$. Dans la seigneurie de Beauport, un moulin à eau

${ }^{73}$ Le premier règlement officiel qui assure aux seigneurs la banalité du moulin à farine qu'ils construisent dans leurs fiefs, paraît être du $1^{\text {er }}$ juil. 1675 (EO, II: 62s.); à la suite d'un arrêt du roi, le 4 juin 1686, tous les seigneurs sont tenus de construire des moulins banaux dans un an: à faute de ce faire, le droit de banalité appartient aux particuliers qui construiront des moulins (EO, I: 255s.).

74 Acte de concession du 20 juillet 1652 (minutier Auber).

$75 \mathrm{On}$ avait réservé pour ce moulin à vent un emplacement d'un demiarpent, sur la pointe du Château-Richer; d'après diverses allusions dans des actes de concession, il serait en opération depuis au moins 1657 .

76 Situé sur un emplacement de 30 perches cédées par Jean Cloutier aux seigneurs de Beaupré, le 24 juil. 1662 (ASQ, Registre A: 282-284); une cession de Macé Gravel, le 13 nov. 1657, atteste l'existence de ce moulin (ASQ, Séminaire, 36: 11).

77 Une transaction du 15 août 1656 établit qu'il y a déjà un moulin dans Argentenay (minutier Audouart), mais les bâtiments sont abandonnés à partir de 1661 . 
existe déjà en $1637^{78}$. La métairie Notre-Dame-des-Anges, dans la seigneurie de ce nom, a son moulin à vent, qui remplace celui que les Jésuites avaient d'abord établi sur la rive droite de la rivière Saint-Charles, au lieu dit $L a$ Vacherie ${ }^{79}$. Dans la ville de Québec, nous connaissons le moulin que Simon Denys de La Trinité a construit en 1658, sur les hauteurs du Cap-aux-Diamants, dans le même style que celui de Notre-Dame-des-Anges ${ }^{80}$, ainsi que le moulin à vent que les Jésuites déclarent, à l'automne de 1663 , sur une pointe de terre près du Collège ${ }^{81}$; dans la banlieue, Jean Bourdon exploite un moulin à vent sur son fief Saint-Jean ${ }^{82}$, mais ce n'est pas là un moulin attaché à sa seigneurie, puisque Bourdon n'y a pas de censitaires; Jean Juchereau de Maure a un moulin à eau sur sa terre Saint-Denys ${ }^{83}$; toutefois, Juchereau de Maure, Bourdon, Denys de La Trinité et les Jésuites ne pouvaient faire farine pour les gens de la ville et de la banlieue, que sur l'autorisation des Cent-Associés, détenteurs de la banalité en vertu de leur seigneurie directe du sol ${ }^{84}$. Les Jésuites de Sillery ont érigé un moulin à vent, sur la falaise qui domine l'anse Saint-Joseph ${ }^{85}$. Sur la rive droite du fleuve, dans la seigneurie de Lauson, il n'existe en 1663 que le moulin à eau construit par François Bissot dit Larivière, dès 1655, sur la terre qu'on lui a concédée: ce moulin a été construit avec l'assentiment formel du seigneur Jean de Lauson

78 Aveu et dénombrement du 22 mars 1659 (ASQ, Faribault, 2: 108).

79 Déclaration des terres, RJ, 47: 260; sur le moulin de La Vacherie, qui est remplacé par celui de Notre-Dame-des-Anges, voir les actes de concession de février 1652 (minutier Audouart).

80 Marché du 30 nov. 1658 (minutier Peuvret de Mesnu). Ce moulin était construit sur la terre de 6 arpents qui appartenait à Denys de La Trinité (terre qui couvrirait aujourd'hui une partie de la citadelle); le 5 juil. 1663, Denys de La Trinité obtiendra 2 arpents, plus bas, au Mont-Carmel, pour y déménager son moulin: c'est là que le représente le plan de Villeneuve en 1685 .

81 Construit sur un emplacement triangulaire qui domine le pied de la Côte-de-la-Fabrique et qui avait été concédé aux Jésuites, le 10 juin 1663, ce moulin est déclaré à l'automne de 1663 (Déclaration des terres, RJ, 47: 258): il était probablement en activité, au moment de notre inventaire, en juin 1663.

82 La présence de ce moulin est signalée dans un bail du 26 juil. 1655 (minutier Audouart) et ce moulin est encore en activité en 1665.

83 Acte de vente du $11^{\text {er }}$ mai 1662 (minutier Audouart).

84 On ne connaît à Bourdon qu'un seul censitaire (établi dans la seigneurie de Saint-François); quant à Juchereau de Maure, à Denys de La Trinité et aux Jésuites de la haute-ville, leurs moulins n'étaient pas édifiés sur des terres concédées en seigneurie. Nous ignorons la nature des conventions faites entre eux et les Cent-Associés; un accord du 22 juil. 1644 (minutier Tronquet) ne nous éclaire que sur un partage des droits des fermiers. 85 Déclaration des terres, RJ, 47: 262. 
et les habitants sont tenus d'y faire moudre leurs grains comme à un moulin banal ${ }^{86}$.

Dans la région des Trois-Rivières, inscrivons un moulin à vent dans le Cap-de-la-Madeleine, seigneurie des Jésuites à l'embouchure de la rivière Saint-Maurice ${ }^{87}$; et un autre moulin à vent, construit vers 1649 par Jacques Leneuf de La Poterie, près des ruines du fort de 1634 , moulin qu'il reconstruit en $1661^{88}$.

Les censitaires de Montréal disposent du moulin à vent construit en 1658 sur le côteau Saint-Louis, pour remplacer l'ancien moulin qu'on avait, dès les débuts, érigé près du fort de Ville-Marie ${ }^{89}$.

Données que résume le tableau suivant: RÉGION DE QUÉBEC

Beaupré

Beauport

Notre-Dame-des-Anges

ville et banlieue de Québec

Sillery

Lauson

RÉGION DES TROIS-RIVIÈrES

Cap-de-la-Madeleine

Trois-Rivières moulin à vent des seigneurs, au ChâteauRicher

moulin à eau des seigneurs, sur la rivière du Sault-à-la-Puce

moulin à eau du seigneur

moulin à vent des seigneurs

moulin à vent exploité par Simon Denys de La Trinité (banalité des Cent-Associés)

moulin à vent exploité par les Jésuites (banalité des Cent-Associés)

moulin à vent exploité par Jean Bourdon (banalité des Cent-Associés)

moulin à eau exploité par Jean Juchereau de Maure (banalité des Cent-Associés) moulin à vent des seigneurs

moulin à eau exploité par François Bissot dit Larivière, au nom du seigneur

moulin à vent des seigneurs

moulin à vent exploité par Jacques Leneuf de La Poterie (banalité des Cent-Associés)

${ }^{86}$ Voir là-dessus, J.-E. Roy, Histoire de la seigneurie de Lauzon, I: 229s.; II : 12-20.

87 Déclaration des terres, RJ, 47: 266. Moulin construit vis-à-vis l'île de La Poterie, dans le fort dit fort du moulin à vent.

88 Déclaration du 23 avril 1661 (minutier Ameau). Ce moulin était construit sur une terre qui relevait directement des Cent-Associés.

89 Voir, en particulier, le bail de ce moulin à Pierre Bessonnet, 20 déc. 1658 (minutier Basset). L'ancien moulin, situé près du fort, ne paraît plus être en activité en 1663 . 
RÉGION DE MONTRÉAL

Montréal

moulin à vent du seigneur

Nous obtenons ainsi un total de 13 moulins à farine, dont neuf sont à vent. Les trois-quarts de ces moulins à farine sont érigés dans la région de Québec, où vivent les $65,1 \%$ de la population totale; la région des Trois-Rivières n'en compte que deux, pour les 15,2\% de la population; Montréal, qui réunit les $19,7 \%$ de la population, n'a qu'un seul moulin. Nous sommes surpris qu'en 1663 si peu de seigneuries aient un moulin à farine: Beaupré, Beauport, Notre-Dame-des-Anges, Sillery, Lauson, le Cap-de-la-Madeleine et Montréal; soit seulement sept seigneuries sur les 47 seigneuries qui nous paraissent occupées en 1663 (fiefs, arrière-fiefs et arrière-arrière-fiefs). Combien les seigneurs pouvaient-ils retirer de ces moulins et combien le fermage des moulins de la ville et banlieue de Québec et des Trois-Rivières pouvait-il rapporter aux Cent-Associés ? Les actes de concession ne précisent pas encore quelle quantité de farine le titulaire du moulin banal pourra retenir pour le droit de mouture; comme les concessions sont faites en conformité avec la Coutume de Paris ${ }^{90}$, on peut supposer que, dès ces débuts, ce droit s'élève au quatorzième; toutefois, de savoir que le seigneur peut retenir le quatorzième de la mouture, ne nous avance guère, puisque nous ignorons tout du rendement de ces moulins à farine, du temps des Cent-Associés.

\section{e - autres droits lucratifs}

La pêche littorale, pratiquée sur un pied industriel et commercial, tombe aussi sous la banalité du seigneur. Les censitaires sont généralement autorisés à pêcher devant leurs concessions pour fins de subsistance, sans que le seigneur ne perçoive un droit; et il en est de même pour la chasse qu'ils peuvent faire à l'intérieur des limites de leurs terres, liberté générale de chasse et de pêche que le gouverneur Lauson confirme en 1652, à condition qu'on n'aille ni chez les voisins ni sur les terres non concédées ${ }^{91}$.

Là où l'exploitation de la pêche dépasse les fins de subsistance, le seigneur prend sa part des profits. Dans la banlieue de Québec, les exploitants doivent remettre aux Cent-Associés un quarteron d'anguilles, c'est-à-dire le vingt-cinquième; au Cap-

${ }^{90}$ Les exceptions ne nous paraissent porter que sur le droit de retrait, que l'on exerce en conformité avec la Coutume de Normandie.

91 Ordonnance du 13 avril 1652, dans RAPQ (1924-25) : 377. 
Rouge, dans la seigneurie de Gaudarville où les Cent-Associés ont concédé des terres avant l'érection en seigneurie, le droit perçu est la trentième anguille; dans la seigneurie de Lauson, les droits varient: c'est tantôt la trentième anguille ou le trentième saumon, l'un et l'autre salés et bien conditionnés, ainsi qu'on l'exige de Jean Bourdon et de Couillart de Lespinay, c'est tantôt la onzième anguille ou le onzième saumon, ou généralement, le onzième poisson, quand cela ne va pas jusqu'au dixième poisson, anguille ou autre; en d'autres cas, c'est un quarteron d'anguilles; dans l'arrière-fief Saint-Vilmé (toujours en Lauson), Pépin et Gaulin devront fournir au seigneur d'Ailleboust, sur la grève de Coulonge ou de Québec, "au choix dud. Seig ${ }^{\mathrm{r}} \mathrm{au}$ tems que ce faict la pesche d'anguille", cinq mille anguilles "fraisches mortes au sel, lavée et bien conditionnée", le seigneur se réservant d'acheter le surplus, aussi salé et bien conditionné, à 20 livres la barrique ${ }^{92}$.

Les institutions religieuses qui font une exploitation importante de la pêche et auxquelles on réserve un monopole dans un lieu précis, sont dispensées de redevances: l'Hôtel-Dieu jouit de ce monopole à l'embouchure de la rivière Saint-Charles, "a l'endroit de [ses] maisons \& Clostures et Jusques au milieu du Canal dud. fleuve Seulement" ${ }^{93}$; en 1651, on lui ajoute le droit de pêcher tout poisson, "le long du fleuve $S^{t}$. Laurent au dessoubs de la coste du cap aux diamants", entre la pêche des Ursulines et celle de la Fabrique de Notre-Dame ${ }^{94}$; les Ursulines ont leur pêche, depuis le 26 octobre 1650, "à la descente du Cap aux Diamants pour aller à Sillery en venant vers le quay de Québec aboutissant en un lieu ou a esté marqué un cèdre" 95 ; la Fabrique de Notre-Dame obtient une pêche, le 1er octobre 1651, depuis la terre de Jacques Maheust en descendant vers l'embouchure de la rivière Saint-Charles ${ }^{96}$. A Sillery, la pêche appartient aux Sauvages chrétiens, titulaires du fief; il est vrai qu'en 1650, les Cent-Associés avaient réservé aux gouverneurs la pêche de la Pointe-à-Puiseaux, sur une largeur de 3 arpents; les Jésuites, administrateurs de Sillery, s'opposèrent à ce privilège; les gouverneurs Lauson, d'Argenson et Davaugour s'en abstinrent et,

92 Acte de concession du 4 juin 1653 (minutier Audouart). La pêche de l'anguille se faisait en septembre et en octobre (RJ, 45: 192).

93 Acte de concession et de mise en possession, 21 mars et 20 nov. 1648 (copie collationnée du 1er oct. 1670), AHD-Q, Terrier des anciens titres, 6b-7a; PTIO, 165.

94 PTIO, 160.

95 PTIO, 93. Le “quay de Québec” est la basse-ville.

96 PTIO, 281. La terre de Jacques Maheust, dans la banlieue du Capaux-Diamants, commençait à 6 arpents de la ligne de circonvallation. 
finalement, Mésy y renoncera formellement en $1664^{97}$. De sorte que, le long du Cap-aux-Diamants, de Sillery à la rivière SaintCharles, une chaîne d'institutions (Sauvages chrétiens, Ursulines, Hôtel-Dieu et Fabrique de Notre-Dame) exploite la pêche en 1663 , sans verser de redevances.

Le seigneur peut trouver une autre source de profit, s'il met à la disposition de censitaires, un lieu où ils puissent en commun faire paître leurs bestiaux. Toutefois, du temps des Cent-Associés, les Communes des régions de Québec et des TroisRivières sont gratuites: à Beaupré, les prés de grève qui sont communs; à Beauport, la Commune d'environ 210 arpents; à Sillery, la Commune de 5 arpents, qui sert à la défense; aux Trois-Rivières, la Commune de 425 arpents. C'est à Montréal seulement que la jouissance en commun est taxée: la Commune de 40 arpents, qui s'étire entre le fleuve et la ville, entraîne une taxe de 6 deniers par habitant, et la prairie qui donne sur la rivière Saint-Pierre coûte 5 sols par année aux censitaires de cet endroit; pour la première, les seigneurs de Montréal ont dû, en théorie, percevoir en 1662 des 32 titulaires d'emplacement urbain la petite somme de 16 sols; pour la prairie, le rapport a pu être d'environ 5 livres.

Inscrivons encore au profit du seigneur, son droit de retrait féodal. Ce droit, habituellement affirmé dans les actes de concession en censive, assure au seigneur la priorité sur tout acquéreur d'une terre déjà concédée; le seigneur a 40 jours pour exercer ce droit et il doit payer "le prix que l'acquereur en a baillé \& payé, \& les loyaux cousts" ${ }^{98}$; ainsi, dans le cas d'une vente à vil prix, le seigneur peut reprendre la terre et la revendre à meilleur prix, de façon à percevoir des lods et ventes plus élevés. Selon la Coutume de Paris, le remboursement, par le seigneur, du premier prix de vente doit se faire dans les 24 heures qui suivent cette vente; c'est pour obtenir plus de facilité en ce domaine, que les seigneurs de Beaupré, en ce qui touche le retrait, dérogent à la Coutume de Paris pour appliquer la Coutume de Normandie. Nous ne connaissons, du temps des Cent-Associés, qu'un seul cas clair et précis de retrait féodal:

97 Voir les documents reproduits dans Scott, Notre-Dame de SainteFoy, I: 524-534.

98 Ferrière, op. cit., I: 60-63. Il ne faut pas confondre retrait féodal et retrait lignager; étant donné la vente d'une terre, le seigneur peut, dans les 40 jours, se porter acquéreur de cette terre: c'est le retrait féodal; le retrait lignager "est un droit par lequel un parent du côté \& ligne duquel l'heritage vendu est échû au vendeur, peut retirer l'heritage des mains de l'acquereur pour le conserver dans la famille"; le parent a un délai d'un an pour exercer ce droit (ibid., I: 272ss). 
à Montréal, Jean Gasteau avait acheté de Gilbert Barbier, le 6 décembre 1660, une terre de 30 arpents pour le prix de 650 livres; le surlendemain, le commandant Chomedey exerce le droit de retrait, au nom de la Société de Notre-Dame: il reprend la terre et en transmet le titre à la veuve d'Ailleboust ${ }^{99}$. Le droit de retrait ne s'applique pas seulement dans le cas d'une vente; il arrive que le seigneur se réserve de reprendre une terre simplement parce qu'il peut y trouver son avantage: lorsqu'il concède une terre à Pierre Masse, le 30 juillet 1645, le seigneur Chavigny de Berchereau se garde le droit de reprendre, dans six ans, la terre avec ses bâtiments, à condition (et c'est bien le moins) de concéder à Masse une autre terre de pareille étendue ${ }^{1}$ : cette condition menaçait de ravaler Masse à un simple rôle de fermier, mais elle ne paraît pas s'être appliquée, puisqu'il fallut quitter les lieux à cause des Iroquois.

Droit lucratif aussi que celui de la corvée, entendant ici non pas le travail que les censitaires doivent faire en commun dans l'intérêt général de la seigneurie (par exemple, l'établissement et l'entretien des chemins), mais le travail accompli pour le profit personnel du seigneur. Sur cette corvée d'ordre personnel, nous n'avons trouvé, au cours de notre période, aucune ordonnance de l'autorité publique, et nous ne connaissons que deux seigneuries où le titulaire soumette le censitaire à cette corvée: Beauport et Saint-Joseph. A Beauport, le seigneur Giffard oblige Noël Langlois, lors de la concession de 1637, à lui fournir chaque année deux journées d'homme, à l'époque des récoltes ${ }^{2}$; de même, en concédant 200 arpents, le 20 juin 1654 , aux associés Zacharie Maheust, René Maheust et Toussaint Giroux, Giffard impose à chacun, sur réquisition du seigneur, deux journées d'homme ${ }^{3}$. Dans sa seigneurie de Saint-Joseph, Guillaume Couillart exige de Rageot dit Lelyonnais (qui occupe une terre depuis 1661) qu'il fauche "en un endroit designé contenant environ deux arpens" (et nous comprenons qu'il s'agit du domaine personnel du seigneur), mais Rageot refuse son titre de concession à cause de cette clause; il sera même prêt en décembre 1663 à abandonner sa terre, à condition que le seigneur le dédommage du travail déjà fait, ce que le seigneur accepte 4 .

99 Acte de vente du 6 déc. 1660 et retrait féodal du 18 déc. 1660 (minutier Basset). La terre est vendue pour le prix de 650 livres; lors du retrait féodal, on convient de payer à Gasteau 650 livres, plus 84 livres 5 sols de dépenses qu'il avait faites, dans l'intervalle, pour sa terre.

1 Acte de concession du 30 juil. 1645 (minutier Tronquet).

2 Acte de concession du 29 juin 1637 (minutier Lespinasse).

3 Acte de concession du 20 juin 1654 (minutier Badeau). 1663).

4 Jugements et délibérations du Conseil souverain, I: 86s. (22 déc. 
Trois seuls cas de corvée seigneuriale personnelle que l'on connaisse du temps des Cent-Associés: la corvée personnelle n'est pas encore un système établi.

Deux autres prérogatives sont au bénéfice du seigneur. Dans les titres de concession qu'il accorde à certains de ses censitaires, le seigneur de Beauport se réserve le droit de faire usage de leurs prairies ${ }^{5}$. Quant au bois qui est sur la terre concédée, le seigneur peut continuer d'en tirer profit: dans la seigneurie de Sillery, au moins deux censitaires ${ }^{6}$ devront "permettre aux sauvages de Sillery", qui sont les seigneurs, "de Coupper et prendre du bois de chauffage et pour autre usage", sans les "molester ou empescher quant et lors qu'ils en auront besoin" 7; dans l'île de Montréal, la Société de Notre-Dame se réserve, sur les terres des habitants, le droit de prendre du bois de chauffage et de construction et l'on précise même que le censitaire devra laisser prendre du bois de chauffage à quiconque présente une permission du gouverneur ${ }^{8}$; dans les premières années d'un établissement, il pouvait être nécessaire que le bois, article essentiel de consommation, devînt pour ainsi dire possession commune, mais l'usage de ce droit par le seigneur pouvait facilement devenir abusif: l'Etat interviendra en 1706, au bénéfice des censitaires de Montréal, pour limiter ce droit ${ }^{\text {. }}$

\section{$\mathrm{f}$ - diverses servitudes des censitaires}

L'acte de concession porte à la charge du censitaire diverses clauses qui limitent de bien des côtés la liberté du censitaire, celui-ci n'étant juridiquement que "concessionnaire" ou dépositaire d'une tranche du sol.

Le censitaire devra tenir feu et lieu, c'est-à-dire avoir, sur sa terre, une maison habitée. Le délai d'installation est parfois déterminé: dans la seigneurie de Beaupré, Lepicard qui se fait concéder une terre, le 7 avril 1646, devra s'y établir dès cette année; pour la terre que Letardif reçoit en 1655, un délai est accordé jusqu'en 1657; Thibault, qui concède une terre à Ouimet en second arrentement, le 8 novembre 1659, accorde même un délai de quatre ans; dans Notre-Dame-des-Anges, dans Coulonge

5 Actes de concession des 19 oct. 1649 (minutier Bermen) et 1er mars 1655 (minutier Badeau).

6 Etienne Denevers, au bord du fleuve, et Guillaume Bonhomme, dans la côte Saint-Michel.

7 Acte de concession du 10 déc. 1660 (minutier Audouart). Sulpice.

8 Voir les divers actes de concession, dans APC, Documents Saint-

9 Ordonnance de Raudot, 2 juil. 1706, EO, II : 263s. 
et dans Saint-Michel, le censitaire doit se bâtir dans "l'an et jour"; dans Sillery, nous connaissons au moins un cas où le censitaire devra commencer à défricher dans un mois à compter du 15 mai et bâtir dans l'an ${ }^{10}$. A Montréal, le censitaire rural s'engage à faire sa demeure dans l'île et non ailleurs ${ }^{11}$, à se construire au plus tôt et à avoir une maison en ville. Là où il y a projet de village, comme dans l'arrière-fief Beaulieu, le seigneur exige que le censitaire se construise dans ce village ${ }^{12}$. La concession d'emplacement dans les villes entraîne la même obligation de "bastir sur ledit emplacement dans l'an et jour, autrement la concession [deviendra] nulle"; Mathieu Huboust, pour sa part, voit ce délai se prolonger jusqu'à un an et demi; de même aux Trois-Rivières, il faut bâtir dans l'an. D'où l'on voit l'importance, lors de l'enquête de 1667-1668, de pouvoir déclarer qu'on a construit une maison sur son emplacement.

Une fois la terre concédée, si le censitaire veut la faire arpenter, c'est lui, au moins dans la seigneurie de Beauport ${ }^{13}$, qui en assumera les frais. Il est tenu de défricher ou, comme on disait alors, de "déserter". Dans Notre-Dame-des-Anges, le censitaire devra cultiver sa terre "en sorte que les dits cens et rentes puissent estre perceus par chacun an"; dans le Cap-de-laMadeleine, on l'oblige simplement, selon la mentalité paternaliste de l'époque, à "cultiver comme un bon père de famille". Il faut en même temps donner du découvert à ses voisins, c'est-à-dire ne pas laisser du bois qui nuise, par l'ombre et l'humidité, à la culture du voisin.

A mesure qu'il fait du défrichement, il est obligé "de clore ses terres", sinon il ne peut prétendre "aucuns domage ny Interests pour les degats quy pourroient faire les bestiaux de ses voisins": obligation constamment rappelée dans les actes de concession de Beaupré, de Notre-Dame-des-Anges et du Cap-dela-Madeleine. Aux Trois-Rivières, cette obligation de clore s'applique même à la palissade de la ville: Cailleteau n'ayant pas travaillé à cette corvée, son emplacement de 240 toises, concédé en 1650, lui fut enlevé et reconcédé en 1651 à Etienne Delafond; Mathurin Baillargeon perdit, pour la même raison, l'emplacement

10 Acte de concession du 23 janv. 1652 (minutier Godet).

11 Le censitaire peut quitter Montréal, mais à condition de remettre les gratifications qu'on lui a accordées; ce qui n'était pas toujours possible: le chirurgien Etienne Bouchard voulait quitter Montréal, mais il aurait été obligé de remettre 1000 livres au seigneur, il n'en sera dispensé qu'en 1667 (actes des 29 avril 1660 et 4 janv. 1667, minutier Basset).

12 Actes de concession des 29 juil. 1658 et 9 fév. 1659 (minutier Audouart).

13 Acte de concession du 19 oct. 1649 (minutier Bermen). 
qu'on lui avait concédé en 1650 : Pierre Dandonneau en deviendra le titulaire en 1652; Gilles Trottier reçut une punition un peu moins lourde: son emplacement de 400 toises fut réduit de moitié ${ }^{14}$.

Le censitaire doit, en outre, souffrir les chemins qui sont nécessaires à l'intérieur de la seigneurie et y travailler, s'il y a lieu. Dans la seigneurie de Beaupré, par exemple, il devra y avoir un chemin de quinze pieds de largeur le long du fleuve et, là où ce sera jugé à propos, un chemin de même largeur entre les "tenants" ou voisins. Dans Notre-Dame-des-Anges, on fera passer un chemin de 2 toises (12 pieds) à travers toutes les concessions, de la rivière Saint-Charles jusqu'à Beauport et, en particulier, un chemin de 2 toises entre les terres de Huppé et de Crevier. Dans Sillery, ceux qui ont droit de pêche vis-à-vis leur concession, devront ensemble, là où il sera jugé bon, faire un chemin pour qu'ils puissent "commodement descendre la coste" ${ }^{15}$.

Autre obligation, qui n'apparaît toutefois que dans les actes de concession de Beauport: "en cas de guerre seront les dits preneurs obligés de ce refugier en la maison Seigneurialle", pour suivre "les ordonnances et les observer" ${ }^{16}$, mais ce n'était pas là une obligation proprement seigneuriale, les habitants étant simplement tenus, selon les ordonnances, de se retirer dans les lieux fortifiés, en cas d'attaque par les Iroquois.

N'est pas non plus une obligation seigneuriale celle de payer la dîme ecclésiale, même si, dans la seigneurie de Beaupré, l'acte de concession précise constamment que le censitaire devra payer "la disme au Curé lorsque le cas y Escherra": c'est que la Compagnie de Beaupré, en faisant une distinction nette entre un revenu qui va au seigneur et un revenu qui appartient au curé, veut dès les débuts jouer, en quelque sorte, le rôle de bras séculier au bénéfice du curé.

Enfin, le censitaire qui désire céder sa terre, subit une dernière servitude: il ne peut vendre sa terre sans l'autorisation du seigneur. C'est du moins une exigence imposée par les titulaires de quatre seigneuries: Beaupré, Notre-Dame-des-Anges, Lauson et Montréal; dans Notre-Dame-des-Anges et dans Lauson, cette permission n'est accordée que s'il y a déjà 10 ou 12 arpents

14 Voir là-dessus, Boucher de La Bruère, La naissance des TroisRivières, 29-31.

15 Acte de concession du 23 janv. 1652 (minutier Godet).

16 Acte de concession du 20 juin 1654 (minutier Badeau). 
en labour. Nous n'avons pas ce qu'il faut pour vérifier si cette exigence a été appliquée dans toute sa rigueur.

\section{Le calendrier seigneurial}

Les droits à payer sont portables et non quérables, c'est-àdire que le seigneur n'a pas à se rendre chez le censitaire pour les percevoir; c'est à ce dernier d'aller les acquitter au manoir: un grand nombre d'actes de concession sont explicites là-dessus.

Pour l'acquittement des droits, qu'il s'agisse de paiement en numéraire ou en nature, le censitaire doit se conformer à un calendrier, qui est modelé sur le calendrier ecclésial. Les dates ne sont pas toutes les mêmes d'une seigneurie à une autre, ni même à l'intérieur d'une seigneurie donnée:

\section{Beaupré}

11 novembre: saint Martin

sauf dans l'arrière-fief Lothainville et dans l'arrière-fief Charny-Lirec, où les censitaires paient les cens et rentes à la Saint-Rémi, chef d'octobre ( $1^{\text {er }}$ octobre); dans le petit arrière-fief Beaulieu, trois dates: certains censitaires paient à la Saint-Michel (29 septembre), d'autres à la SaintEtienne (26 décembre) et d'autres à la Saint-Jean-l'Evangéliste (27 décembre); un concessionnaire en sous-arrentement paie sa rente à la Fabrique du Château-Richer, le 2 juillet, fête de la Visitation, mais ce cas n'entre pas, à proprement parler, dans ce calendrier seigneurial

Beauport

11 novembre: saint Martin

toutefois, avant 1655 (sauf dans un cas de 1637), le censitaire devait acquitter ses droits à la Noël, 25 décembre

Notre-Dame-des-Anges

26 décembre: saint Etienne

Sault-au-Matelot

11 novembre: saint Martin

Fabrique de

Notre-Dame-de-Québec 11 novembre: saint Martin

Coulonge

$1^{\text {er }}$ octobre: saint Rémi

sauf pour les chapons, que le censitaire devait présenter le 27 décembre, jour de la Saint-Jean-l'Evangéliste

Saint-Michel

Saint-François

Sillery
25 décembre: Noël

27 décembre: saint Jean l'Evangéliste

27 décembre: saint Jean l'Evangéliste

sauf les censitaires de la côte Saint-Michel, qui paient à la Toussaint ( $1^{\mathrm{er}}$ novembre); et Nicolas Chaigneau (dont la concession est antérieure à l'érection en seigneurie) : il paie à la Saint-Martin (11 novembre) 
Lauson $1^{\text {er }}$ octobre: saint Rémi

sauf les censitaires Eustache Lambert, Denys Duquet, les Ursulines et quelques autres censitaires qui paient à la Saint-Martin (11 novembre); Georges Cadoret doit présenter ses anguilles à la Saint-Martin (11 novembre); Guillaume Couture doit présenter les siennes à la Saint-Michel (29 septembre); dans l'arrière-fief Saint-Vilmé, on présente ses chapons à la Saint-Jean-l'Evangéliste (27 décembre), mais l'argent des cens et rentes à la Saint-Rémi ( ${ }^{\mathrm{er}}$ octobre)

Cap-de-la-Madeleine

11 novembre: saint Martin

îles du Saint-Maurice $1^{\text {er }}$ octobre: saint Rémi

il s'agit des terres qui relèvent directement des Cent-Associés au point de vue des cens et rentes

Trois-Rivières: ville et banlieue $\quad 1^{\text {er }}$ octobre: saint Rémi

il s'agit ici des terres qui relèvent directement des Cent-Associés au point de vue des cens et rentes; signalons que les emplacitaires de la terre de Pachiriny paient à la Saint-Thomas, apôtre (21 décembre), mais ce cas de sous-arrentement n'entre pas ici dans notre calendrier seigneurial

Montréal: campagne

11 novembre: saint Martin

$\mathrm{Si}$, au lieu de procéder selon l'ordre géographique des seigneuries, nous suivons l'ordre du calendrier seigneurial, nous obtenons le tableau suivant:

29 septembre, saint Michel

certains censitaires de l'arrière-fief Beaulieu, dans l'île d'Orléans droit de pêche de Guillaume Couture, dans Lauson

$1^{\text {er }}$ octobre, saint Rémi

les censitaires des arrière-fiefs Lothainville et Charny-Lirec, dans le fief Beaupré

les censitaires de Coulonge (sauf pour la présentation des chapons)

les censitaires de Lauson (à l'exception des quelques-uns)

les censitaires des îles du Saint-Maurice, de la ville et banlieue des TroisRivières

$1^{\text {er }}$ novembre, Toussaint

les censitaires de la côte Saint-Michel, dans Sillery

11 novembre, saint Martin

les censitaires de Beaupré, sauf ceux des arrière-fiefs Lothainville, Charny-Lirec et Beaulieu

les censitaires de Beauport (sauf exceptions)

les censitaires de la ville de Québec (ce sont ceux du Sault-au-Matelot et de la Fabrique de Notre-Dame)

Nicolas Chaigneau, dans la seigneurie de Sillery 
certains censitaires de Lauson, dont Georges Cadoret qui doit acquitter ses droits de pêche, ce jour-là

les censitaires du Cap-de-la-Madeleine

les censitaires de la campagne de Montréal

25 décembre, Nö̈l

certains censitaires de Beauport

les censitaires de Saint-Michel

26 décembre, saint Etienne

certains censitaires de l'arrière-fief Beaulieu, dans l'île d'Orléans

les censitaires de Notre-Dame-des-Anges

\section{7 décembre, saint Jean l'Evangéliste}

un censitaire de l'arrière-fief Beaulieu

présentation des chapons dans Coulonge

les censitaires de Saint-François

les censitaires de Sillery (sauf ceux de la côte Saint-Michel et Nicolas

Chaigneau)

présentation des chapons dans l'arrière-fief Saint-Vilmé (Lauson).

Si maintenant nous calculons la superficie en arpents que recouvrent ces sept dates du calendrier seigneurial, nous obtenons, sur un total de 105666,1 arpents, les pourcentages qui suivent:

29 septembre, saint Michel

ler octobre, saint Rémi

ler novembre, Toussaint

11 novembre, saint Martin

25 décembre, Noël

26 décembre, saint Etienne

28 décembre, saint Jean l'Evangéliste

\begin{tabular}{|c|c|}
\hline superficie & $\%$ \\
\hline 692 & 0,7 \\
\hline 27184,6 & 25,7 \\
\hline 1425 & 1,3 \\
\hline 66914,5 & 63,3 \\
\hline 1232,5 & 1,2 \\
\hline 5105 & 4,8 \\
\hline 3112,5 & 3 \\
\hline
\end{tabular}

La Saint-Martin, 11 novembre, est donc la date seigneuriale la plus en usage, dans le paiement des droits: elle recouvre près des deux tiers de la superficie en censive de 1663; elle est suivie, mais de loin, par la Saint-Rémi, ler octobre, qui en recouvre le quart. Les autres fêtes ne comptent que pour bien peu dans l'ensemble: la Toussaint, 1er novembre, ne s'applique qu'à un groupe de censitaires de Sillery; la Saint-Etienne, 26 décembre, n'est la règle que dans Notre-Dame-des-Anges; la Saint-Jean-l'Evangéliste, 27 décembre, vaut seulement pour une partie des censitaires de Sillery; Noël, 25 décembre, ne prédomine que dans Beauport; la Saint-Michel, 29 septembre, si importante dans d'autres domaines, ne recouvre ici que moins d'un pour cent du territoire. 
Pourquoi telle fête plutôt que telle autre? Pour les terres qui relèvent d'eux, les Cent-Associés ont choisi la Saint-Rémi, sans que nous puissions expliquer cette préférence. De même, nous cherchons en vain un rapport de ces dates avec les fêtes patronales des seigneurs ou avec les noms des seigneuries, ou encore avec les fêtes de dévotion particulière: ni les Jésuites ni la Société de Notre-Dame, par exemple, n'ont adopté une fête qui leur soit propre; le 29 septembre n'a été retenu ni dans la seigneurie de Saint-Michel ni dans la côte Saint-Michel. Ces dates du calendrier seigneurial n'ont que deux points en commun: toutes (sauf la Saint-Rémi) sont des fêtes d'obligation, à cette époque; et elles sont toutes dans le dernier tiers de l'année: en octobre et en novembre, les moissons et les pêcheries sont terminées, les chapons et les poules ont fini leur engraissement. Passé le temps de la cueillette, vient celui de payer son dû: du 29 septembre au 27 décembre, les censitaires défilent vers le manoir, mais ce sont le premier octobre et le 11 novembre que l'activité de la perception est particulièrement importante.

\section{Une approximation des revenus bruts de 1662}

Comme nous l'avons fait pour les cens, rentes, lods et ventes, procédons d'une façon théorique, faute de livres de compte, et tentons d'esquisser un tableau de ce que les terres en censive et les emplacements ont pu rapporter aux seigneurs, à la fin de 1662: (Voir page suivante).

Selon notre approximation, les revenus bruts de 1662 se chiffreraient à 2889 livres 7 sols 3 deniers:

$\begin{array}{lrr}\text { cens } & 235 \text { livres } 19 \text { sols } 3 \text { deniers } & 8,2 \% \\ \text { rente (chapons inclus) } & 1818 \text { livres } 2 \text { sols } & 62,9 \% \\ \text { lods et ventes } & 817 \text { livres } 10 \text { sols } & 28,3 \% \\ \text { autres } & 17 \text { livres } 16 \text { sols } & 0,6 \%\end{array}$

Approximation fort incomplète, puisqu'elle ne recouvre ni les revenus tirés en cens et rentes des emplacements de la ville de Québec (ceux qui relèvent des Cent-Associés), ni ceux qui proviennent des moulins à farine et des pêcheries; toutefois, s'ils étaient connus, ces revenus des pêcheries ne s'appliqueraient (les Cent-Associés mis à part) qu'à un seul seigneur, celui de Lauson; les revenus tirés des moulins à farine ne modifieraient l'actif que de huit seigneuries. De sorte que, croyons-nous, notre total de 2889 livres 7 sols 3 deniers donne, de la situation générale des revenus seigneuriaux de 1662 une image assez fidèle. Nous constatons ici que c'est la rente qui rapporte le plus: 
Fiefs et arrière-fiefs

Beaupré

Compagnie de Beaupré

ar.-f. Repent.-Villiers

ar.-f. Repentigny

ar.-f. Lothainville

ar.-f. Argentenay

ar.-f. Charny-Lirec

ar.-ar.-f. La Chevalerie

ar.-f. Beaulieu

Beauport

ar.-f. Beaumarchais

Notre-Dame-des-Anges

Saint-Joseph

Saint-Ignace

Québec: ville

Québec: banlieue

Coulonge

Saint-Michel

Saint-François

Sillery

ar.-f. Sainte-Ursule

Gaudarville

Lauson

ar.-f. Sainte-Anne

ar.-f. Saint-Vilmé

Cap-de-la-Madeleine

île de la Trinité

l'Islet

île Saint-Christophe

Ile-aux-Cochons

Trois-Rivières: ville

Trois-Rivières: banlieue

Montréal: ville

Montréal: campagne cens

rente

lods
et ventes

autres total

liv. s. d. liv. s. d. liv. s. d. liv. s. d. liv. s. d. $\begin{array}{lll}905 & 9 & 6\end{array}$

$\begin{array}{lll}55 & 3 & 2\end{array}$

$\begin{array}{lll}24 & 3 & 6\end{array}$

$\begin{array}{lll}52 & 8 & 8\end{array}$

117

$34412 \quad 6$

$\begin{array}{lll}39 & 0 & 0\end{array}$

$\begin{array}{lll}51 & 2 & 3\end{array}$

$12 \quad 0 \quad 0^{*} \quad 68 \quad 2 \quad 0$

$\begin{array}{lll}3 & 12 & 0\end{array}$

$\begin{array}{lll}183 & 17 & 8\end{array}$

$1913 \quad 4$

$\begin{array}{lll}2 & 0 & 2\end{array}$

$85 \quad 0 \quad 0$

$\begin{array}{lll}51 & 3 & 10\end{array}$

$\begin{array}{lll}15 & 9 & 0\end{array}$

$\begin{array}{lll}10 & 0 & 0\end{array}$

$\begin{array}{lll}2 & 2 & 0\end{array}$

$\begin{array}{lll}168 & 17 & 1\end{array}$

$\begin{array}{lll}14 & 0 & 4\end{array}$

$\begin{array}{lll}43 & 14 & 6\end{array}$

$\begin{array}{lll}175 & 14 & 4\end{array}$

$\begin{array}{lll}13 & 7 & 0\end{array}$

$\begin{array}{lll}9 & 0 & 0\end{array}$

$\begin{array}{lll}187 & 0 & 0\end{array}$

$\begin{array}{lll}1 & 5 & 0\end{array}$

$\begin{array}{lll}0 & 1 & 0\end{array}$

4112

$\begin{array}{lll}25 & 0 & 0\end{array}$

$\begin{array}{lll}60 & 9 & 9\end{array}$

$\begin{array}{lll}77 & 6 & 5\end{array}$

$\begin{array}{lll}77 & 6 & 5\end{array}$

$\begin{array}{lll}58 & 15 & 0\end{array}$

$\begin{array}{rrrrrrrrr}143 & 5 & 0 & 0 & 16 & 0^{* *} & 150 & 0 & 0\end{array}$

$\begin{array}{llllllllll}2 & 1 & 8 & 5 & 0 & 0^{* * *} & 34 & 14 & 1\end{array}$

* Cette somme de 12 livres est l'équivalent de 8 journées d'homme que Giffard exige en corvée; nous évaluons la journée d'homme à 30 sols, qui est le salaire d'un homme à tout faire, à Sainte-Anne-de-Beaupré en 1662 (comptes de Fabrique, dans RAPQ (1967): 178ss).

** Ces 16 sols sont ce que rapporte, en principe, la Commune en 1662.

*** Ces 5 livres sont les droits perçus, en principe, pour l'usage des prairies de la rivière Saint-Pierre. 
$62,9 \%$; c'est en même temps un revenu stable. Les lods et ventes, qui ne sont qu'un revenu casuel, comptent en 1662 pour un pourcentage élevé, $28,3 \%$, qui illustre bien l'instabilité des censitaires. Le cens est d'importance négligeable, mais il est quand même plus élevé que nous ne l'avions d'abord cru, étant donné sa nature de symbole.

Des seigneuries qui rapportent le plus, celle de Beaupré vient en tête: 905 livres pour les terres qui relèvent de la Compagnie de Beaupré; 345 livres pour les terres de l'arrière-fief Charny-Lirec; le total de Beaupré serait encore plus élevé, si nous connaissions les revenus des deux moulins à farine. Quelques autres seigneuries sont d'un certain rapport: le Cap-de-laMadeleine, 187 livres; Montréal, 185 livres; Notre-Dame-desAnges, 184 livres; Lauson, 176 livres; Sillery, 169 livres.

Si nous distribuons ces maigres revenus de 1662 entre les divers titulaires de la terre en seigneurie, nous obtenons la répartition suivante:

\section{Jésuites 17}

Charles de Lauson-Charny 18

les Cent-Associés 19

la veuve de Jean de Lauson, fils 20

Jean Rosée, fils 21

Société de Notre-Dame de Montréal 22

les Sauvages chrétiens de Sillery

Charles Aubert de La Chesnaye 23

les héritiers Berruyer 23

Antoine Cheffault de La Regnardière 23

Jacques Duhamel 23

la veuve de François Fouquet 23

François de Laval, vicaire apostolique ${ }^{23}$

Robert Giffard 24

Jean-Baptiste Legardeur de Repentigny 25

la veuve de Chavigny de Berchereau 26

la veuve de Louis Lauson de La Citière 27

la veuve de Louis d'Ailleboust 28

Charles Legardeur de Villiers 29

Ursulines de Québec ${ }^{30}$

Jacques Leneuf de La Poterie 31

Jean Juchereau de La Ferté 32

Nicolas Juchereau de Saint-Denys 33

Guillaume Couillart 34

Charles Legardeur de Tilly ${ }^{35}$

Jean Bourdon 36

Hôtel-Dieu de Québec 37

$\begin{array}{ccc}\text { livres } & \text { sols } & \text { deniers } \\ 375 & 8 & 10 \\ 348 & 4 & 6 \\ 275 & 6 & 0 \\ 228 & 3 & 0 \\ 226 & 5 & 0 \\ 184 & 14 & 1 \\ 168 & 17 & 1 \\ 113 & 2 & 6 \\ 113 & 2 & 6 \\ 113 & 2 & 6 \\ 113 & 2 & 6 \\ 113 & 2 & 6 \\ 113 & 2 & 6 \\ 68 & 2 & 0 \\ 51 & 15 & 1 \\ 51 & 2 & 3 \\ 43 & 14 & 6 \\ 35 & 16 & 0 \\ 27 & 11 & 7 \\ 27 & 7 & 4 \\ 25 & 0 & 0 \\ 19 & 10 & 0 \\ 19 & 10 & 0 \\ 19 & 3 & 4 \\ 10 & 0 & 0 \\ 2 & 2 & 0 \\ 2 & 0 & 2\end{array}$

17 Ils retirent 183 livres 17 sols 8 deniers de Notre-Dame-des-Anges, 
Dans ces revenus seigneuriaux de 1662 , les Jésuites viennent en tête avec 375 livres 8 sols 10 deniers ${ }^{38}$, suivis de près par un individu, le prêtre Charles de Lauson-Charny, avec 348 livres 4 sols 6 deniers; les Cent-Associés ne viennent qu'au troisième rang, avec 275 livres 6 sols : c'est qu'ils n'arrentent pas les terres. Des 27 seigneurs ici énumérés, 14 ne touchent pas 100 livres de revenus; parmi ceux-ci, Robert Giffard fait petite figure avec 68 livres 2 sols, et il faut en dire autant de la veuve d'Ailleboust, de Guillaume Couillart, de Jean Bourdon; les Communautés de femmes ne retirent encore presque rien de leurs seigneuries: les Ursulines de Québec, 27 livres 7 sols 4 deniers; l'Hôtel-Dieu de Québec, un ridicule 2 livres 2 deniers.

187 livres du Cap-de-la-Madeleine et 4 livres 11 sols 2 deniers de l'île Saint-Christophe.

$18 \mathrm{Il}$ retire 344 livres 12 sols 6 deniers de son arrière-fief CharnyLirec et 3 livres 12 sols de son arrière-fief Beaumarchais.

19 Cette somme provient d'emplacements de Québec (85 livres), de terres de la banlieue de Québec (51 livres 3 sols 10 deniers), de l'île de la Trinité (1 livre 5 sols), de l'Islet (1 sol), d'emplacements de la ville des Trois-Rivières (60 livres 9 sols 9 deniers) et des terres de sa banlieue (77 livres 6 sols 5 deniers).

20 Elle retire 62 livres 8 sols 8 deniers de son arrière-fief Lothainville et 175 livres 14 sols 4 deniers de Lauson. Beaupré.

$21 \mathrm{~A}$ cause des deux huitièmes qu'il détient dans la Compagnie de

${ }_{22}$ Soit 150 livres d'emplacements de la ville et 34 livres 14 sols 1 denier des terres rurales.

23 Pour un huitième de la Compagnie de Beaupré.

24 Il retire 68 livres 2 sols de sa seigneurie de Beauport.

25 Il retire 27 livres 11 sols 7 deniers de son arrière-fief Repentigny-

Villiers et 24 livres 3 sols 6 deniers de son arrière-fief Repentigny.

26 Elle retire 51 livres 2 sols 3 deniers de son arrière-fief Beaulieu.

27 Elle retire 43 livres 14 sols 6 deniers de Gaudarville.

28 Elle retire 11 livres 7 sols de son arrière-fief Argentenay, 15 livres

9 sols de Coulonge et 9 livres de son arrière-fief Saint-Vilmé. Villiers.

29 Il retire 27 livres 11 sols 7 deniers de son arrière-fief Repentigny-

30 Elles retirent 14 livres 4 deniers de leur arrière-fief Sainte-Ursule et 13 livres 7 sols de leur arrière-fief Sainte-Anne.

$31 \mathrm{Il}$ retire 25 livres de l'île aux Cochons.

32 Il retire 19 livres 10 sols de son arrière-arrière-fief La Chevalerie.

33 Voir la note précédente.

34 Il retire 19 livres 3 sols 4 deniers de sa seigneurie de Saint-Joseph.

$35 \mathrm{Il}$ retire 10 livres de sa seigneurie de Saint-Michel.

36 Il retire 2 livres 2 sols de sa seigneurie de Saint-François.

37 L'Hôtel-Dieu retire cette somme de la seigneurie de Saint-Ignace.

38 De fait, il faudrait ajouter à ce montant 168 livres 17 sols 1 denier que les Jésuites retirent des terres de Sillery, dont ils sont alors les administrateurs, au nom des Sauvages chrétiens. 
Le total de 2889 livres 7 sols 3 deniers, formé du revenu brut de plus de 400 terres (soit un revenu moyen de 7 livres par terre), démontre, indépendamment des dépenses encourues par le seigneur et que nous ne connaissons pas, qu'à la fin du régime des Cent-Associés, cette première génération des seigneurs n'en est pas encore une de rentiers du sol. Il resterait à déterminer à partir de quel moment les censitaires arriveront à faire vivre leur seigneur, seulement en s'acquittant de leurs obligations. 